\title{
Large Scale Geometries of Infinite Strings
}

\author{
Bakh Khoussainov \\ Department of Computer Science \\ The University of Auckland \\ Auckland, New Zealand \\ Email: bmk@cs.auckland.ac.nz
}

\author{
Toru Takisaka \\ Research Institute for Mathematical Sciences \\ Kyoto University \\ Kyoto, Japan \\ Email: takisaka@kurims.kyoto-u.ac.jp
}

\begin{abstract}
We introduce geometric consideration into the theory of formal languages. We aim to shed light on our understanding of global patterns that occur on infinite strings. We utilise methods of geometric group theory. Our emphasis is on large scale geometries. Two infinite strings have the same large scale geometry if there are colour preserving bi-Lipschitz maps with distortions between the strings. Call these maps quasi-isometries. Introduction of large scale geometries poses several questions. The first question asks to study the partial order induced by quasi-isometries. This partial order compares large scale geometries; as such it presents an algebraic tool for classification of global patterns. We prove there is a greatest large scale geometry and infinitely many minimal large scale geometries. The second question is related to understanding the quasiisometric maps on various classes of strings. The third question investigates the sets of large scale geometries of strings accepted by computational models, e.g. Büchi automata. We provide an algorithm that describes large scale geometries of strings accepted by Büchi automata. This links large scale geometries with automata theory. The fourth question studies the complexity of the quasi-isometry problem. We show the problem is $\Sigma_{3}^{0}$-complete thus providing a bridge with computability theory. Finally, the fifth question asks to build algebraic structures that are invariants of large scale geometries. We invoke asymptotic cones, a key concept in geometric group theory, defined via model-theoretic notion of ultra-product. Partly, we study asymptotic cones of algorithmically random strings thus connecting the topic with algorithmic randomness.
\end{abstract}

\section{INTRODUCTION}

Our goal is to introduce geometric considerations into the theory of formal languages. We emphasise the study of large scale geometries of infinite strings. Our hope is to shed light on our understanding of global large scale patterns that occur on infinite strings. Our motivation comes from geometric group theory, a cutting edge research area in group theory linked with geometry, topology, automata, logic, probability, complexity, etc.

In geometric group theory, an important concept is that of quasi-isometry between metric spaces. Let $\mathcal{M}_{1}=\left(M_{1}, d_{1}\right)$ and $\mathcal{M}_{2}=\left(M_{2}, d_{2}\right)$ be metric spaces.

Definition I.1. A function $f: M_{1} \rightarrow M_{2}$ is called an $(A, B)$-quasi-isometry from $\mathcal{M}_{1}$ to $\mathcal{M}_{2}$, where $A \geqslant 1$ and $B \geqslant 0$, if for all $x, y \in M_{1}$ we have

$$
(1 / A) \cdot d_{1}(x, y)-B \leqslant d_{2}(f(x), f(y)) \leqslant A \cdot d_{1}(x, y)+B,
$$

978-1-5090-3018-7/17/\$31.00 (C)2017 IEEE and for all $y \in M_{2}$ there exists an element $x \in M_{1}$ such that $d_{2}(y, f(x)) \leqslant A$.

Note that when $B=0$, the mapping becomes bi-Lipschitz (and hence continuous). Thus, a quasi-isometry $f: \mathcal{M}_{1} \rightarrow$ $\mathcal{M}_{2}$ behaves like a bi-Lipschitz map with distortion $B$ between the metric spaces. For instance, the metric spaces $\mathbb{Z}$ (integers) and $\mathbb{R}$ (reals) with their natural metric are quasi-isometric Informally, two metric spaces $\mathcal{M}_{1}$ and $\mathcal{M}_{2}$ are quasi-isometric if these spaces (such as $\mathbb{Z}$ and $\mathbb{R}$ ) look the same from far away. The quasi-isometry relation forms an equivalence relation on the class of all metric spaces.

Studying quasi-isometry invariants of groups turned out to be crucial in solving many problems in group theory [6] [7] [8]. Therefore, finding quasi-isometry invariants has become an important theme in geometric group theory. Examples of quasi-isometry invariants are: being virtually nilpotent, virtually free, hyperbolic, having polynomial growth rate, being finitely presentable, having decidable word problem, asymptotic cones [4] [5] [8].

In formal language theory and logic, one of the main objects are infinite strings $\alpha$ over finite alphabets $\Sigma$. These objects are somewhat boring from a geometry point of view. The strings $\alpha$ possess the natural metric inherited from the set of natural numbers $\omega$. The quasi-isometry type of $\omega$ is the metric space $\mathbb{R}_{\geqslant 0}$ of all positive reals. So, from a quasi-isometry view point, $\omega$ viewed as a metric space is also somewhat uneventful. However, one crucial difference from the setting in geometric group theory is that the metric spaces $\alpha$ are coloured. Namely, for every position $i \in \omega$ in $\alpha$, the colour of the position $i$ is $\sigma$ when $\alpha(i)=\sigma$. These observations suggest that the notions and methods of geometric group theory (e.g. quasi-isometry) can be applied to coloured metric spaces. Here we investigate quasi-isometries of coloured metric spaces with strings $\alpha$ as our primary objects, thus initiating the study of large scale patterns on strings.

A coloured metric space $\mathcal{M}$ is a tuple $(M ; d, C)$, where $(M ; d)$ is the underlying metric space with metric $d, C$ is a colour function $C: M \rightarrow 2^{\Sigma}$, and $\Sigma$ is a finite set of colours that we call an alphabet. If $\sigma \in C(m)$ then we say that $m$ has colour $\sigma$. As mentioned above, infinite strings $\alpha$ are coloured metric spaces of the form $(\omega ; d, \alpha)$, where $d$ is the natural metric (so, $d(i, j)=|i-j|$ ) and $\alpha: \omega \rightarrow \Sigma$ is the colour function. Every element in $\alpha$ has a unique colour. We always 
assume that the cardinality $|\Sigma|$ of $\Sigma$ is at least 2 .

We denote the set of all infinite strings over $\Sigma$, considered as coloured metric spaces, by $\Sigma^{\omega}$. We now adapt Definition I.1 for coloured metric spaces.

Definition I.2. Assume we are given coloured metric spaces $\mathcal{M}_{1}=\left(M_{1} ; d_{1}, C_{1}\right)$ and $\mathcal{M}_{2}=\left(M_{2} ; d_{2}, C_{2}\right)$. A colour preserving quasi-isometry from $\left(M_{1} ; d_{1}\right)$ into $\left(M_{2} ; d_{2}\right)$ is called a quasi-isometry from $\mathcal{M}_{1}$ into $\mathcal{M}_{2}$.

We write $\mathcal{M}_{1} \leqslant Q I \mathcal{M}_{2}$ if there is a quasi-isometry from $\mathcal{M}_{1}$ into $\mathcal{M}_{2}$. For metric spaces the relation $\leqslant_{Q I}$ is an equivalence relation, and hence it is symmetric. In contrast, for coloured metric spaces the relation $\leqslant_{Q I}$ is not symmetric. For instance, the mapping $f: \omega \rightarrow \omega$ defined as $f(i)=2 i$ is a quasi-isometry from the string $0^{\omega}=00000 \ldots$ into the string $(01)^{\omega}=010101 \ldots$. There is no quasi-isometric mapping in the opposite direction.

If $\mathcal{M}_{1} \leqslant Q I \mathcal{M}_{2}$ and $\mathcal{M}_{2} \leqslant Q I \mathcal{M}_{1}$ then we write this by $\mathcal{M}_{1} \sim_{Q I} \mathcal{M}_{2}$. The relation $\sim_{Q I}$ is an equivalence relation in the class of all coloured metric spaces, in particular on the set $\Sigma^{\omega}$. Formally:

Definition I.3. The equivalence classes of $\sim_{Q I}$ are called the quasi-isometry types or, equivalently, the large scale geometries. For the set $\Sigma^{\omega}$, we define $\Sigma_{Q I}^{\omega}=\Sigma^{\omega} / \sim_{Q I}$. Denote the large scale geometry of string $\alpha$ by $[\alpha]$. Thus, $\Sigma_{Q I}^{\omega}=\left\{[\alpha] \mid \alpha \in \Sigma^{\omega}\right\}$.

Non-symmetry of $\leqslant Q I$ on coloured metric spaces allows us to compare large scale geometries of these spaces, and consider the partial order $\leqslant_{Q I}$ on the quasi-isometry types. Importantly, the partial order $\leqslant_{Q I}$ can be restricted to the large scale geometries of coloured metric spaces over a fixed underlying metric space (e.g. $\omega$ with its natural metric). In this sense the quasi-isometry on coloured metric spaces is a much refined version of the usual quasi-isometry relation on unclouded metric space. This is because the quasi-isometry type of every uncoloured metric space trivialises to a singleton.

Introduction of large scale geometries and the quasiisometry relation $\leqslant Q I$ poses a vast amount of natural questions. The contribution of this paper consists of investigating the following questions:

The first question is concerned with understanding the partial order $\leqslant_{Q I}$ on the set $\Sigma_{Q I}^{\omega}$ of all large scale geometries. The order presents an algebraic tool aimed at classification of global patterns that occur on strings. In Section [I] we investigate properties of this partial order. Among several results, we prove that the order has the greatest large scale geometry, infinite chains and infinite anti-chains. We show that there are infinitely many minimal large scale geometries.

The second question is related to understanding the quasiisometry relation $\sim_{Q I}$. This can be done by either restricting the relation $\sim_{Q I}$ on various subclasses of infinite strings or by simplifying the definition of quasi-isometry. In Section III-A we restrict the relation $\sim_{Q I}$ to eventually periodic words, and give a full description of quasi-isometry types in this class. Section II-B gives a intuitively more easier yet equivalent definition of the relation $\leqslant_{Q I}$ that we call component-wise reducibility. We also give more refined version of $\leqslant_{Q I}$, colourequivalence, that implies quasi-isometry. It is shown that colour-equivalence is strictly stronger than quasi-isometry. It is natural to ask if quasi-isometric maps between strings can be replaced with order preserving quasi-isometric embeddings. We give a negative answer to this question; however, we prove that the answer is positive modulo $\sim_{Q I}$ equivalence relation.

The third question is related to describing sets of large scale geometries. We call such sets atlases. Let $L$ be a language of infinite strings. One considers the atlas $[L]$ of all large scale geometries of strings in $L$. So, the question is related to understanding the atlas $[L]$ given a description of $L$. In particular, a natural question is if one can decide that $\left[L_{1}\right]=\left[L_{2}\right]$ given descriptions of languages $L_{1}$ and $L_{2}$. In Section IV] we use Büchi automata as a description language and provide a full characterisation of the atlases defined by Büchi recognisable languages. As a consequence, we show that for Büchi automata recognisable languages $L_{1}$ and $L_{2}$ there is a linear time algorithm for deciding the equality of the atlases $\left[L_{1}\right]$ and $\left[L_{2}\right]$. This contrasts with the PSPACE completeness of the equality problem for Büchi languages. This part of the work links large scale geometries with automata theory and complexity theory.

The fourth question addresses the complexity of quasiisometry relation on infinite strings in terms of arithmetical hierarchy, thus connecting the topic with computability theory. Recall that isometry is colour preserving and distance preserving map. So, quasi-isometry relation is weaker than isometry relation. Hence, one might expect that quasi-isometry is easier to detect than the isometry. We prove that the quasi-isometry relation on computable infinite strings is $\Sigma_{3}^{0}$-complete. This is in contrast to $\Pi_{1}^{0}$-completeness of the isometry relation on computable strings. These are explained in Section $\mathrm{V}$.

The fifth question asks how one encodes large scale geometries of coloured metric spaces $\alpha$ into "limit" structures. For this, we define structures obtained from "looking at $\alpha$ from far away". Dries and Wilkie [4], using ultra-filters, formalised this intuitive notion through the concept of asymptotic cone. Their work gave a logical context to Gromov's work in [6] [8]. We invoke the concept of asymptotic cone and study relationship between large scale geometries of infinite strings and their asymptotic cones. In Section VI we prove theorems akin to results on asymptotic cones in geometric group theory, and we show that asymptotic cones of Martin-Löf random strings coincide when scaling factors are computable. These results bridge the topic of this paper with algorithmic randomness and model theory.

\section{THE PARTIAL ORDER $\left(\Sigma_{Q I}^{\omega}, \leqslant Q I\right)$}

\section{A. Basic Properties}

The quasi-isometry relation $\leqslant_{Q I}$ naturally induces the partially ordered set $\left(\Sigma_{Q I}^{\omega}, \leqslant Q I\right)$ on the set of all large scale geometries $[\alpha]$. Denote this partial order by $\Sigma_{Q I}^{\omega}$ thus identifying it with its domain. Say that $[\beta]$ covers $[\alpha]$ if $[\alpha] \neq[\beta]$, $[\alpha] \leqslant_{Q I}[\beta]$, and for all $[\gamma]$ such that $[\alpha] \leqslant_{Q I}[\gamma] \leqslant_{Q I}[\beta]$ 
we have $[\gamma]=[\alpha]$ or $[\gamma]=[\beta]$. An element is an atom if it covers a minimal element.

Proposition II.1. The partial order $\Sigma_{Q I}^{\omega}$ has the following properties: (1) There exists a greatest element; (2) There exist at least $|\Sigma|$ minimal elements; (3) There exist at least $|\Sigma|$. $(|\Sigma|-1) / 2$ atoms.

Proof. Assume that $\Sigma=\left\{\sigma_{1}, \ldots, \sigma_{k}\right\}$. For part (1), we claim that $\alpha=\left(\sigma_{1} \ldots \sigma_{k}\right)^{\omega}$ is the greatest element in $\Sigma_{Q I}$. Indeed, take any $\beta \in \Sigma^{\omega}$. We write $\alpha$ as $v_{0} v_{1} \ldots$ where each $v_{i}$ is $\sigma_{1} \ldots \sigma_{k}$. Define $f: \beta \rightarrow \alpha$ such that $f$ maps any position $n$ (in $\beta$ ) to the position $k_{n}$ in the portion $v_{n}$ of the string $\alpha$ such that $\beta(n)=\alpha\left(k_{n}\right)$. For part (2), consider the quasiisometry types $\left[\sigma_{i}^{\omega}\right]$. These form minimal elements in $\Sigma_{Q I}^{\omega}$. Indeed, if $\alpha \leqslant_{Q I} \sigma_{i}^{\omega}$, then each element of $\alpha$ has colour $\sigma_{i}$. For part (3), consider $\sigma_{i}\left(\sigma_{j}\right)^{\omega}$, where $i \neq j$. Clearly, $\sigma_{j}^{\omega} \leqslant Q I$ $\sigma_{i}\left(\sigma_{j}\right)^{\omega}$. Moreover, for any $\beta \neq \sigma_{j}^{\omega}$ if $\beta \leqslant Q I \sigma_{i}\left(\sigma_{j}\right)^{\omega}$ then $\beta$ finitely many positions of $\beta$ are coloured with $\sigma_{i}$, and all other positions are coloured with $\sigma_{j}$. Hence, $\beta \sim_{Q I} \sigma_{i}\left(\sigma_{j}\right)^{\omega}$. Thus, we have found at least $k \cdot(k-1) / 2$ of the atoms.

A quasi-isometry $g: \alpha \rightarrow \beta$ can produce cross-overs, e.g., pairs $n$ and $m$ with $n<m$ but $g(m)<g(n)$. The definition of quasi-isometry does not obviously prohibit large crossovers. Nevertheless, the following lemma shows that there is a uniform bound on cross-overs.

Lemma II.2 (Small Cross Over Lemma). Consider a quasiisometry map $g: \alpha \rightarrow \beta$. There is a constant $C \leqslant 0$ such that for all $n<m$ we have $g(m)-g(n) \geqslant C$.

Proof. Let $n<m$ be given and suppose we have $g(m)-$ $g(n)<C$ for some $C \leqslant 0$. The goal is to provide a lower bound for $C$. Define $q=\min \left(g^{-1}(p) \cap[m+1, \infty)\right)$ and $p=\min (g([m+1, \infty)) \cap[g(n)+1, \infty))$. Then, we obtain

$$
\begin{aligned}
d(g(n), p) & \geqslant(1 / A) \cdot d(n, q)-B \geqslant(1 / A) d(n, m)-B \\
& \geqslant(1 / A)(1 / A)(d(g(n), g(m))-B)-B \\
& \geqslant-C / A^{2}-\left(\left(A^{2}+1\right) /\left(A^{2}\right)\right) B .
\end{aligned}
$$

We have $g([m, q]) \subset[0, g(n)] \cup[p, \infty)$ by our definition of $p$ and $q$. Note $g(m) \in[0, g(n)]$ and $g(q) \in[g(p), \infty)$. Hence, there exists $r_{C} \in[m, q-1]$ such that $g\left(r_{C}\right) \in[0, g(n)]$ and $g\left(r_{C}+1\right) \in[g(p), \infty)$, which means

$$
d(g(n), p) \leqslant d\left(g\left(r_{C}\right), g\left(r_{C}+1\right)\right) \leqslant A+B .
$$

From these inequalities we have a lower bound on $C$.

Lemma II.3. Let $f: \alpha \rightarrow \beta$ be a quasi-isometric mapping. Then there exists constants $A^{\prime}$ and $B^{\prime}$ such that for all positions $x, y \in \beta$ with $x \leqslant y$ we have

$$
\begin{gathered}
\left(1 / A^{\prime}\right) \cdot d_{2}(x, y)-B^{\prime} \leqslant \\
d_{1}\left(\min f^{-1}([x, y]), \max f^{-1}([x, y])\right) \leqslant A^{\prime} \cdot d_{2}(x, y)+B^{\prime} .
\end{gathered}
$$

Proof. The inequality in the definition of quasi-isometry implies:

$$
(1 / A) \cdot d_{2}(f(n), f(m))-(B / A) \leqslant d_{1}(n, m)
$$

$$
\leqslant A \cdot d_{2}(f(n), f(m))+A B .
$$

For given $x \leqslant y \in \beta$, let $M_{x y}=\max f^{-1}([x, y])$ and $m_{x y}=\min f^{-1}([x, y])$. Then we have $d_{1}\left(m_{x y}, M_{x y}\right) \leqslant$ $A \cdot d_{2}\left(f\left(m_{x y}\right), f\left(M_{x y}\right)\right)+A B \leqslant A \cdot d_{2}(x, y)+A B$. For the lower bound of $d_{1}\left(m_{x y}, M_{x y}\right)$, let $M_{x y}^{\prime}=\max ([x, y] \cap f(\alpha))$ and $m_{x y}^{\prime}=\min ([x, y] \cap f(\alpha))$. Then due to the last condition of quasi-isometricity it holds that $d_{2}\left(y, M_{x y}^{\prime}\right) \leqslant 2 A$ and $d_{2}\left(x, m_{x y}^{\prime}\right) \leqslant 2 A$. Also from Lemma II.2 we have

$$
d_{2}\left(f\left(M_{x y}\right), M_{x y}^{\prime}\right) \leqslant-C
$$

and

$$
d_{2}\left(f\left(m_{x y}\right), m_{x y}^{\prime}\right) \leqslant-C .
$$

Hence $d_{2}\left(f\left(m_{x y}\right), f\left(M_{x y}\right)\right) \geqslant d_{2}(x, y)-4 A+2 C$. In summary, for any $x \leqslant y \in \beta$ it holds that

$$
\begin{aligned}
& (1 / A) \cdot d_{2}(x, y)-(4 A+B-2 C) / A \\
\leqslant & d_{1}\left(m_{x y}, M_{x y}\right) \leqslant A \cdot d_{2}(x, y)+A B .
\end{aligned}
$$

Set $A^{\prime}=A$ and $B^{\prime}=(4 A+B-2 C) / A+A B$.

Corollary II.4. Let $f: \alpha \rightarrow \beta$ be a quasi-isometry. There is $a C>0$ so that $\left|f^{-1}(y)\right|<C$ for all $y \in \beta$.

\section{B. Structure theorems}

Elementary properties of the partial order $\Sigma_{Q I}^{\omega}$ are in Proposition II.1. Now we provide several structure theorems describing algebraic properties of $\Sigma_{Q I}^{\omega}$.

Theorem II.5. The partial order $\Sigma_{Q I}^{\omega}$ has a chain $\left(\alpha_{n}\right)_{n \in \mathbb{Z}}$ of the type of integers, that is $\forall n \in \mathbb{Z}\left[\begin{array}{lll}\alpha_{n} & <_{Q I} & \alpha_{n+1}\end{array}\right]$. Furthermore, the partial order $\Sigma_{Q I}^{\omega}$ has a countable antichain.

Proof. We prove the first part. Take two distinct element in $\Sigma$, say 0 and 1 , respectively. Let

$$
\alpha_{n}= \begin{cases}(01)^{2^{n}}(011)^{2^{n}} \ldots\left(01^{2^{k}}\right)^{2^{n}} \ldots & (n \geqslant 0) \\ (01)\left(01^{2^{2^{-n}}}\right) \ldots\left(01^{\left(2^{k}\right)^{2^{-n}}}\right) \ldots & (n<0)\end{cases}
$$

The idea is the following. The string $\alpha_{0}$ is of the form:

$$
(01)(011)(01111) \ldots\left(01^{2^{k}}\right) \ldots
$$

Let us call the substrings $\left(01^{2^{k}}\right)$ blocks of $\alpha_{0}$. The above definition tells us that $\alpha_{1}$ is obtained from $\alpha_{0}$ by doubling each block of $\alpha_{0} ; \alpha_{-1}$ is obtained from $\alpha_{0}$ by removing every other block. This is propagated to all $\alpha_{n}$ 's.

We show that $\alpha_{n}<_{Q I} \alpha_{n+1}$ for all $n \geqslant 0$. For negative $n$, the proof is similar. To see $\alpha_{n} \leqslant Q I \alpha_{n+1}$, consider the mapping which sends an interval $\left(01^{2^{k+1}}\right)$ in $\alpha_{n}$ to $\left(01^{2^{k}}\right)^{2}$ in $\alpha_{n+1}$ in an obvious injective way. This mapping is a quasiisometry from $\alpha_{n}$ into $\alpha_{n+1}$.

To see that the converse is not true, assume that $g$ is a quasi-isometry from $\alpha_{n+1}$ into $\alpha_{n}$. Then using Lemma I.3 and Lemma $\amalg .2$ one sees that $g$ needs to be strictly monotone on almost every point of $\alpha_{n+1}$ with colour 0 ; that is, we need to have $M \in \mathbb{N}$ such that for all $m, m^{\prime}>M$ and $m>m^{\prime}$ we have $\alpha_{n+1}(m)=\alpha_{n+1}\left(m^{\prime}\right)=0 \Rightarrow g(m)>g\left(m^{\prime}\right)$. 
But this is not possible; indeed, let $m_{i, n} \in \omega$ be the position of $i$-th 0 in $\alpha_{n}$. If $m_{i, n+1}>M$ and $g\left(m_{i, n+1}\right)=$ $m_{j, n}$, then from monotonicity and injectivity we should have $d\left(g\left(m_{i, n+1}\right), g\left(m_{i+k, n+1}\right)\right) \geqslant m_{j+k, n}-m_{j, n}$ for each $k \in \mathbb{N}$. For any $i$ and $j$ we can easily verify

$$
\lim _{k \rightarrow \infty} \frac{m_{j+k, n}-m_{j, n}}{m_{i+k, n+1}-m_{i, n+1}}=+\infty,
$$

which means we cannot have any bound $A$ as in Definition 1. This is contradiction, and hence there is no quasi-isometry from $\alpha_{n+1}$ into $\alpha_{n}$.

Now we prove the second part. Consider the following sequence of strings $\beta_{n}, n \in \omega$ :

$$
\beta_{n}=010^{2^{n}} 1^{2^{n}} 0^{3^{n}} 1^{3^{n}} \ldots 0^{k^{n}} 1^{k^{n}} \ldots
$$

We claim that this sequence forms an anti-chain in $\Sigma_{Q I}^{\omega}$. Take any $n, m \in \mathbb{N}$ and suppose $\beta_{n} \leqslant Q I \beta_{m}$ via $f$. It suffices to show $n=m$.

Let $A_{n^{\prime}, k^{\prime}}$ be the " $k^{\prime}$-th block" of zeros in $\beta_{n^{\prime}}$, i.e.

$$
A_{n^{\prime}, k^{\prime}}=\left[2 \sum_{i=0}^{k^{\prime}-1} i^{n^{\prime}}, 2 \sum_{i=0}^{k^{\prime}-1} i^{n^{\prime}}+\left(k^{\prime}\right)^{n^{\prime}}-1\right]
$$

Then by easy argument we can show that there exists $k, l \in \mathbb{N}$ such that (1) $f\left(A_{n, k}\right) \subseteq A_{m, l}$, and (2) for every $k^{\prime} \in \mathbb{N}$, we have $f\left(A_{n, k^{\prime}+k}\right) \subseteq A_{m, k^{\prime}+l}$. Intuitively, these say that from some point $f$ maps $\beta_{n}$ in "block by block" manner without vacancy. For quasi-isometricity of $f$ we should have an upper and positive lower bound of the rate

$$
\frac{\left|A_{n, k^{\prime}+k}\right|}{\left|A_{m, k^{\prime}+l}\right|}=\frac{\left(k^{\prime}+k\right)^{n}}{\left(k^{\prime}+l\right)^{m}}
$$

with respect to $k^{\prime}$, since otherwise we do not have any bound $A$ as in Definition 1. Clearly $n=m$ is the only case that satisfies this condition.

The trivial large scale geometries $\left[0^{\omega}\right]$ and $\left[1^{\omega}\right]$, as noted above, are minimal elements of $\Sigma_{Q I}^{\omega}$. The next theorem shows that there are non-trivial minimal large scale geometries.

Theorem II.6. Let $\left\{a_{n}\right\}_{n \in \mathbb{N}}$ be an unbounded nondecreasing sequence. Then the large scale geometry of the string $\alpha=0^{a_{0}} 1^{a_{1}} 0^{a_{2}} 1^{a_{3}} \ldots 0^{a_{2 k}} 1^{a_{2 k+1}} \ldots$ is a minimal element in the partial order $\Sigma_{Q I}^{\omega}$.

Proof. Let $I_{n}$ be the interval that corresponds to $p^{a_{n}}(p \in$ $\{0,1\})$ in $\alpha$, that is,

$$
I_{n}=\left[\sum_{k=0}^{n-1} a_{k},\left(\sum_{k=0}^{n} a_{k}\right)-1\right] .
$$

Suppose $\beta \leqslant_{Q I} \alpha$ via $f, A, B$. We claim that $\beta$ is of the form $v w_{0}^{\prime} w_{1} w_{1}^{\prime} w_{2} w_{2}^{\prime} \ldots w_{n} w_{n}^{\prime} \ldots$, where $w_{n}$ and $w_{n}^{\prime}$ are sequences for which we have constants $A^{\prime}, B^{\prime}, D$, and $n_{0}$ such that for all $n$ we have $\left|w_{n}^{\prime}\right| \leqslant D,\left(1 / A^{\prime}\right) \cdot a_{n_{0}+n}-B^{\prime} \leqslant\left|w_{n}\right| \leqslant$ $A^{\prime} a_{n_{0}+n}+B^{\prime}$ and $w_{n}=0^{\left|w_{n}\right|}$ if $n_{0}+n$ is even or $w_{n}=1^{\left|w_{n}\right|}$ if $n_{0}+n$ is odd. If the claim is true, then $\beta={ }_{Q I} \alpha$.
Let $M_{n}=\max f^{-1}\left(I_{n}\right)$ and $m_{n}=\min f^{-1}\left(I_{n}\right)$. From Lemma II.3, there exist $A^{\prime \prime}$ and $B^{\prime \prime}$ such that

$$
\left(1 / A^{\prime \prime}\right) \cdot a_{n}-B^{\prime \prime} \leqslant M_{n}-m_{n} \leqslant A^{\prime \prime} a_{n}+B^{\prime \prime}
$$

for each $n$. Then there are numbers $n_{0}$ and $D>0$ such that for all $n \geqslant n_{0}$ we have $m_{n+2}>M_{n}$ and $-D \leqslant m_{n+1}-$ $M_{n} \leqslant 1$. Indeed, let $D=B-A(C-1)$ and $n_{0}$ satisfies $a_{n_{0}} \geqslant A^{\prime \prime}\left(-2 A C+3 \max \left\{B, B^{\prime \prime}\right\}\right)+2$, where $C$ is a constant such that $f(y)-f(x) \geqslant C$ for all $x<y$ (Lemma II.2). Then $m_{n+1}-M_{n} \leqslant 1$ holds since otherwise there exists $x \in \mathbb{N}$ such that $M_{n}<x<m_{n+1}$ which should be mapped to a point other than $I_{n} \cup I_{n+1}$, and we can find $x \in\left[M_{n}, m_{n+1}-1\right]$ such that $d(f(x), f(x+1))>D \geqslant A+B$, which contradicts the quasi-isometricity of $f$. For a lower bound, if $m_{n+1}<M_{n}$ then $f\left(M_{n}\right)-f\left(m_{n+1}\right) \geqslant C$ due to Lemma II.2, and hence

$$
\begin{aligned}
m_{n+1}-M_{n} & =-d\left(m_{n+1}, M_{n}\right) \\
& \geqslant-A \cdot d\left(f\left(m_{n+1}\right), f\left(M_{n}\right)\right)-B \\
& =A \cdot\left(f\left(M_{n}\right)-f\left(m_{n+1}\right)\right)-B \\
& \geqslant A C-B>-D .
\end{aligned}
$$

We also have

$$
\begin{gathered}
m_{n+2}-M_{n}= \\
\left(m_{n+2}-M_{n+1}\right)+\left(M_{n+1}-m_{n+1}\right)+\left(m_{n+1}-M_{n}\right) \\
\geqslant 2 A C-2 B+\left(1 / A^{\prime \prime}\right) \cdot a_{n+1}-B^{\prime \prime}>1 .
\end{gathered}
$$

Now for each $n \geqslant n_{0}$ let $J_{n}=\left[M_{n-1}+1, m_{n+1}-1\right]$ and $J_{n}^{\prime}=\left[m_{n+1}, M_{n}\right]$ if $m_{n+1}<M_{n}$, or otherwise $J_{n}^{\prime}=\phi$. Let $w_{n}$ and $w_{n}^{\prime}$ be the strings that corresponds to $J_{n_{0}+n}$ and $J_{n_{0}+n}^{\prime}$, respectively. Then $\beta$ is of the form $v w_{0}^{\prime} w_{1} w_{1}^{\prime} w_{2} w_{2}^{\prime} \ldots$, and by the inequality above

$$
\begin{gathered}
\left|w_{n}\right|=m_{n_{0}+n+1}-M_{n_{0}+n-1}-1 \geqslant \\
\left(1 / A^{\prime \prime}\right) \cdot a_{n_{0}+n}+2 A C-2 B-B^{\prime \prime}
\end{gathered}
$$

Also, $\left|w_{n}\right| \leqslant M_{n_{0}+n}-m_{n_{0}+n}+1 \leqslant A^{\prime \prime} a_{n_{0}+n}+B^{\prime \prime}+1$. Hence letting $B^{\prime}=\max \left\{-2 A C+2 B+B^{\prime \prime}, B^{\prime \prime}+1\right\}$ and $A^{\prime}=A^{\prime \prime}$ and we have a proof.

Corollary II.7. The partially ordered set $\Sigma_{Q I}^{\omega}$ possesses uncountably many minimal elements.

Consider the string $\alpha=0^{n_{0}} 1^{m_{0}} 0^{n_{1}} 1^{m_{1}} \ldots$ from $\{0,1\}^{\omega}$, where $n_{i}, m_{i} \geqslant 1$. Call the substrings $0^{n_{i}}$ and $1^{m_{i}}$ the 0 blocks and 1-blocks, respectively. Define:

- $\mathcal{X}(0)=\{[\alpha] \mid$ in $\alpha$ all the lengths of 0 -blocks are universally bounded $\}$,

- $\mathcal{X}(1)=\{[\alpha] \mid$ in $\alpha$ the lengths of all 1-blocks are universally bounded $\}$,

- $\mathcal{X}(u)=\{[\alpha] \mid$ in $\alpha$ there is no universal bound on the lengths of both 0 -blocks and 1-blocks $\}$,

- $\mathcal{X}(b)=\{[\alpha] \mid$ in $\alpha$ the lengths of both 0-blocks and 1-blocks are universally bounded $\}$.

Upward closed sets are called filterers, and downward closed sets ideals. We use this terminology in the next theorem.

Theorem II.8. The sets $\mathcal{X}(0), \mathcal{X}(1), \mathcal{X}(u), \mathcal{X}(b)$ satisfy the following: 
1) The sets $\mathcal{X}(0)$ and $\mathcal{X}(1)$ are filters.

2) The set $\mathcal{X}(u)$ is an ideal.

3) The set $\mathcal{X}(b)$ is the singleton $\left\{\left[(01)^{\omega}\right]\right\}$.

Proof. We prove that $\mathcal{X}(0)$ is a filter. Assume that $\alpha \in \mathcal{X}(0)$ and $\alpha \leqslant{ }_{Q I} \beta$ through a quasi-isometry $f$ with constants $A$ and $B$. We need to show that $\beta \in \mathcal{X}(0)$. Assume that the length of 0 -blocks in $\beta$ is unbounded. Let $K$ be the length of the largest 0 -block in $\alpha$. Now, take a 0-block $\beta(i) \ldots \beta\left(i+n_{0}\right)$ in $\beta$, where $n_{0}$ is sufficiently large. We give bound on the value of $n_{0}$ below. Since $f$ is a quasi-isometry, there exists a $\beta\left(i_{0}\right)$ that belongs to the block such that $\beta\left(i_{0}\right)$ has a preimage $\alpha\left(j_{0}\right)$ and $\beta\left(i_{0}\right)$ is within distance $A$ from the center of the 0-block $\beta(i) \ldots \beta\left(i+n_{0}\right)$. The length of the block in $\alpha$ that contains $\alpha\left(j_{0}\right)$ is bounded by $K$, hence the string

$$
\alpha\left(j_{0}-K-1\right) \ldots \alpha\left(j_{0}\right) \ldots \alpha\left(j_{0}+K+1\right)
$$

must contain 1 . The length of this interval is $2 K+2$. There exists a $C$ such that $f$-image of all intervals of length $2 K+2$ is contained in the intervals in $\beta$ of length at most $C$. So, it must be the case that $n_{0} \leqslant C$. Hence $\beta \in \mathcal{X}(0)$. The same proof shows that $\mathcal{X}(1)$ is a filter.

The proof of Part 2 is similar to the proof above.

For the last part for all the strings $\alpha, \beta$ if in both the lengths of 0-blocks and 1-blocks are universally bounded, then $\alpha$ and $\beta$ are quasi-isometric to each other since they are colourequivalent. They are also quasi-isometric to $(01)^{\omega}$.

Corollary II.9. For all $\alpha \in \mathcal{X}(0), \beta \in \mathcal{X}(1), \gamma \in \Sigma^{\omega}$, if $\alpha \leqslant_{Q I} \gamma$ and $\beta \leqslant_{Q I} \gamma$ then $\gamma \sim(01)^{\omega}$.

Consider the join operation $\oplus$ that, given $\alpha$ and $\beta$, produces the string $\alpha \oplus \beta=\alpha(0) \beta(0) \alpha(1) \beta(1) \ldots$ The operation is often used (e.g., in computability and complexity theory) to produce the least upper bounds. It turns out this operation is not well-behaved with respect to the large scale geometries. Indeed, consider the string $\alpha=010011 \ldots(0)^{2^{n}}(1)^{2^{n}} \ldots$ and the string $\beta=101100 \ldots(1)^{2^{n}}(0)^{2^{n}} \ldots$ Then $[\alpha \oplus \beta]=$ $\left[(01)^{\omega}\right]$. But, $\left.\left[(01)^{\omega}\right] \neq[\alpha],\left[(01)^{\omega}\right)\right] \neq[\beta]$, and $[\alpha]=[\beta]$.

Even though the operation $\oplus$ is not well-behaved with respect to $\sim_{Q I}$-classes, the operation can still be useful in constructing counter-examples as shown below.

Corollary II.10. The sets $\mathcal{X}(0), \mathcal{X}(1)$ are not ideals.

Proof. Consider the strings $\beta=010011 \ldots 0^{n} 1^{n} \ldots$ and $\alpha=$ $\beta \oplus 1^{\omega}$. It is clear that $\alpha \in \mathcal{X}(0)$. It is also easy to see that $\beta \leqslant \alpha$. However, $\beta \notin \mathcal{X}(0)$.

Corollary II.11. Both $\mathcal{X}(0)$ and $\mathcal{X}(1)$ have countable chains and anti-chains. In addition, $\mathcal{X}(u)$ has an infinite anti-chain consisting of minimal elements.

Proof. The chain constructed in Theorem $\amalg .5$ is in $\mathcal{X}(0)$. Hence, both $\mathcal{X}(0)$ and $\mathcal{X}(1)$ have countable chains. Let $\beta_{n}$ be the sequence in the proof of Theorem II.5 Then in the same manner as in the proof of Theorem II.5 we can show that $\left\{\beta_{n} \oplus 1^{\omega}\right\}_{n \in \mathbb{N}} \subset \mathcal{X}(0)$ is an anti-chain. Similarly $\left\{\beta_{n} \oplus 0^{\omega}\right\}_{n \in \mathbb{N}} \subset \mathcal{X}(1)$ is an anti-chain. The minimal elements constructed in Theorem II.6 are in $\mathcal{X}(u)$.

\section{REFINING QUASI-ISOMETRY}

The relation $\leqslant_{Q I}$ could be analysed in several ways. One is to restrict $\leqslant_{Q I}$ to a particular class $K$ of infinite strings, and describe the partial order $\leqslant_{Q I}$ restricted to large scale geometers of strings from $K$. In Section $\amalg I-A$, we fully describe the partial order $\leqslant_{Q I}$ restricted to the class of eventually periodic words. The second way is to refine the definition of $\leqslant_{Q I}$ and study its implications. Section $\amalg$ II-B provides an equivalent, more intuitive, characterisation of quasi-isometry called component wise reducibility.

\section{A. Eventually periodic spaces}

For a string $\alpha$ (that might be finite) consider the set of all colours in $\alpha: C l(\alpha)=\{\sigma \in \Sigma \mid \exists i(\alpha(i)=\sigma)\}$. Write $u \sqsubseteq v$ if $C l(u) \subseteq C l(v)$. We easily get the following:

Lemma III.1. If $f: \alpha \rightarrow \beta$ is a quasi-isometry then $C l(\alpha) \subseteq$ $C l(\beta)$. So, if $\alpha \sim_{Q I} \beta$ then $C l(\alpha)=C l(\beta)$.

A particularly simple strings are eventually periodic:

Definition III.2. Metric space $\alpha \in \Sigma^{\omega}$ is eventually periodic if there are finite words $x, u \in \Sigma^{\star}$ such that $\alpha=x u u u \ldots$. Call $u$ the period of $\alpha$.

Let $\alpha=x u^{\omega}$ be eventually periodic word. We assume that $C l(u) \subseteq C l(x)$ as we can change the prefix $x$ to $x u$. With this assumption, we have the following theorem:

Theorem III.3. For eventually periodic words $\alpha, \beta$ we have $\alpha \leqslant Q I \beta$ iff there are $x, y, u, v \in \Sigma^{\star}$ such that $\alpha=x u^{\omega}$, $\beta=y v^{\omega}, C l(x) \subseteq C l(y)$, and $C l(u) \subseteq C l(v)$.

Proof. If $\alpha \leqslant_{Q I} \beta$ then it is easy to select finite strings $x, y, u, v$ that satisfy the statement of the theorem.

Assume that there are $x, y, u, v \in \Sigma^{\star}$ such that $\alpha=x u^{\omega}$, $\beta=y v^{\omega}, C l(x) \subseteq C l(y)$ and $C l(u) \subseteq C l(v)$. Construct a quasi-isometry $f$ from $\alpha$ to $\beta$ as follows. First, map the prefix $x$ into prefix $y$ so that colours are preserved. Secondly, we consider the set $X=\left\{x_{1}, \ldots, x_{k}\right\}$ of all distinct colours that appear in $u$. Now map $x_{i}$ coloured position in the $k^{t h}$ copy of $u$ in the string $\alpha$ into the $x_{i}$ coloured position in the $k^{t h}$ copy of $v$ in the string $\beta$. Set $A=\max \{|x|,|y|,|u|,|v|\}$ and $B=A$. It is clear that $f$ preserves colours and the quasi-isometry inequality between the distances $d(x, y)$ and $d(f(x), f(y))$ with constants $A, B$ as required.

Let $P_{1}(\Sigma)$ be the set of all non-empty subsets of $\Sigma$. Consider the following partial order on the domain:

$$
\mathcal{X}=\left\{(A, B) \mid A, B \in P_{1}(\Sigma) \text { and } A \supseteq B\right\},
$$

where the partial order on $\mathcal{X}$ is the component-wise inclusion. From the theorem above, we obtain the a full description of the partial order $\leqslant Q I$ restricted to large scale geometers of eventually periodic strings.

Corollary III.4. The partial order $\leqslant_{Q I}$ restricted to the set $E P=\{[\alpha] \mid \alpha$ is eventually periodic string over $\Sigma\}$ is isomorphic to the partial order $\mathcal{X}$. 
Let $f: \alpha \rightarrow \beta$ be a quasi-isometric map, and let $P$ be a property. Say that $f$ is eventually $P$ if there is an $i$ such that $f$ restricted to the suffix $\alpha_{i}=\alpha(i) \alpha(i+1) \alpha(i+2) \ldots$ satisfies $P$. A quasi-isometry $f$ does not need to be eventually order preserving map; neither $f$ needs to be eventual embedding (that is, eventually injective map). Now we show that if $\alpha$ and $\beta$ are eventually periodic words, then $f$ can be computable eventually order preserving injective map.

Theorem III.5. For eventually periodic strings $\alpha$ and $\beta$ such that $\alpha \leqslant_{Q I} \beta$ there exists a computable quasi-isometric map $f_{\alpha}: \alpha \rightarrow \beta$ which is eventually order preserving and injective.

Proof. Consider $\alpha=x u u u \ldots$ and $\beta=y v v v \ldots$... We assume that $C l(x) \subseteq C l(y)$ and $C l(u) \subseteq C l(v)$. Our desired mapping $f_{\alpha}$ maps $x$ into $y$ by preserving colours. (That is where $f_{\alpha}$ need not be order preserving embedding). So, it suffices to construct a computable quasi-isometric embedding from $\alpha_{1}=$ $u^{\omega}$ to $\beta_{1}=v^{\omega}$. Let $X=\left\{x_{1}, \ldots, x_{k}\right\}$ be the set of all colours that appear in $\alpha_{1}$, and hence in $\beta_{1}$. Let $a_{i}$ be the number of times that colour $x_{i}$ appears in $v$. Clearly $a_{i} \geqslant 1$. Consider the new string $v_{1}$ obtained by writing $v$ exactly $a_{1}+\ldots+a_{n}$ times, that is, $v_{1}=(v)^{a_{1}+a_{2}+\ldots+a_{n}}$. There exists an embedding $f^{\prime}$ from $u$ into $v_{1}$ that preserves colours and the order. Just like in the theorem above, we can propagate this mapping $f^{\prime}$ to a colour preserving embedding $f_{\alpha}$ from $\alpha_{1}$ into $\beta_{1}$. The map $f_{\alpha}: \alpha \rightarrow \beta$ thus built is a desired function.

\section{B. Componentwise Reducibility}

We formulate an equivalent more intuitive condition for quasi-isometry between coloured metric spaces.

Definition III.6. Say $\alpha$ is componentwise reducible to $\beta$, written $\alpha \leqslant_{C R} \beta$, if we can write $\alpha=u_{1} u_{2} \ldots$ and $\beta=$ $v_{1} v_{2} \ldots$ such that $u_{i} \sqsubseteq v_{i}$ for each $i$ and $\left|u_{i}\right|,\left|v_{i}\right|$ are uniformly bounded by a constant $C$. Call these presentations of $\alpha$ and $\beta$ witnessing partitions.

It is clear that $\alpha \leqslant_{C R} \beta$ implies $\alpha \leqslant_{Q I} \beta$ : any colourpreserving map that maps each interval $u_{i}$ in $\alpha$ to $v_{i}$ in $\beta$ is quasi-isometry. Showing the converse is not trivial. The main difficulty is showing that $\leqslant_{C R}$ is transitive.

Definition III.7. An atomic crossing map is a function $f$ : $\alpha \rightarrow \beta$ of the following form: we have $\left\{a_{i}, b_{i}\right\}_{i \in I}$, where $I$ is an at most countable index set such that $\exists C \forall i\left[\left(a_{i}<b_{i}<\right.\right.$ $\left.\left.a_{i+1}\right) \wedge\left(b_{i}-a_{i} \leqslant C\right)\right]$ and

$$
f(a)= \begin{cases}b_{i} & \left(a=a_{i}\right) \\ a_{i} & \left(a=b_{i}\right) \\ a & \text { (otherwise) }\end{cases}
$$

Clearly, every atomic crossing map is bijective. The next result is a step towards transitivity of $\leqslant_{C R}$.

Proposition III.8. Any quasi-isometry $f: \alpha \rightarrow \beta$ can be decomposed into the following form:

$$
\alpha \stackrel{f_{1}}{\longrightarrow} \gamma_{1} \stackrel{f_{2}}{\longrightarrow} \gamma_{2} \stackrel{f_{3}}{\longrightarrow} \beta
$$

where $f_{1}$ is a monotonic injection, $f_{2}$ is a monotonic surjection, $f_{3}$ is a bijection, and $f_{1}, f_{2}, f_{3}$ are all quasi-isometric. Furthermore, $f_{3}$ can be decomposed into the following form for some $n \geqslant 1$ :

$$
\gamma_{2} \stackrel{g_{1}}{\longrightarrow} \delta_{1} \stackrel{g_{2}}{\longrightarrow} \ldots \stackrel{g_{n}}{\longrightarrow} \beta,
$$

where each $g_{k}$ is an atomic quasi-isometry.

Proof. Let $f: \alpha \rightarrow \beta$ be a quasi-isometry. First we decompose it to $\alpha \stackrel{f^{\prime}}{\longrightarrow} \gamma_{2} \stackrel{f_{3}}{\longrightarrow} \beta$, where $f^{\prime}$ is monotonic and $f_{3}$ is a bijection. By Small Cross Over Lemma we have a bound $D<$ 0 such that $f(m)-f(n) \geqslant D$ for all $n<m$. Let $\left\{\left(n_{i}, m_{i}\right)\right\}_{i \in I}$ be the set of all pairs of natural numbers such that $n_{i}<m_{i}$ and $f\left(m_{i}\right)-f\left(n_{i}\right)=D$, where $I$ is an at most countable index set that depends on $f$. We define $\tilde{\beta}$ as the sequence that is same as $\beta$ except that colours of the position $n_{i}$ and $m_{i}$ are swapped for each $i \in I$ : that is,

$$
\tilde{\beta}(n)= \begin{cases}\beta\left(m_{i}\right) & \left(n=n_{i}\right) \\ \beta\left(n_{i}\right) & \left(n=m_{i}\right) \\ \beta(n) & (\text { otherwise })\end{cases}
$$

Notice that $n_{i}, m_{i}, n_{j}$ and $m_{j}$ are all distinct element for distinct $i, j \in I$, and hence the above definition is welldefined. Also let $\tilde{f}$ be a function that executes this swapping, i.e. $\tilde{f}\left(m_{i}\right)=n_{i}, \tilde{f}\left(n_{i}\right)=m_{i}$ and $\tilde{f}(n)=n$ otherwise. It is easy to show that $\tilde{f} \circ f$ is a QI map from $\alpha$ to $\tilde{\beta}$ such that $\tilde{f} \circ f(m)-\tilde{f} \circ f(n) \geqslant D+1$ for all $n<m$. Iterating this procedure $(-D)$ times we get $\gamma_{2}$ and a bijective QI map $g: \beta \rightarrow \gamma_{2}$ such that $g \circ f$ is monotonic. let $f^{\prime}=g \circ f$ and $f_{3}=g^{-1}$. From the construction it is clear that $g^{-1}$ is a finite composition of certain atomic crossing functions.

Now we decompose $f^{\prime}$ to $\alpha \stackrel{f_{1}}{\longrightarrow} \gamma_{1} \stackrel{f_{2}}{\longrightarrow} \gamma_{2}$, where $f_{1}$ is a monotonic injection and $f_{2}$ is a monotonic surjection. For $\gamma_{2}=c_{1} c_{2} \ldots$, let $\gamma_{1}=\left(c_{1}\right)^{n_{1}}\left(c_{2}\right)^{n_{2}} \ldots$, where $n_{i}=$ $\max \left\{1,\left|f_{1}^{-1}\left(c_{i}\right)\right|\right\}$. Let $f_{1}$ be a map that sends each interval $f^{\prime}\left(c_{i}\right)$ in $\alpha$ to $\left(c_{i}\right)^{n_{i}}$ in $\gamma_{1}$ in an obvious injective, monotonic way. Also let $f_{2}$ be a map that sends each $\left(c_{i}\right)^{n_{i}}$ in $\gamma_{1}$ to $c_{i}$ in $\gamma_{2}$. This is the desired decomposition.

Corollary III.9. If $\alpha \leqslant_{Q I} \beta$, then there is $\beta^{\prime} \sim_{Q I} \beta$ so that $\alpha \leqslant Q I \beta^{\prime}$ via strictly monotonic quasi-isometry.

Proof. The strings $\gamma_{1}$ is a desired $\beta^{\prime}$ as $\beta^{\prime}$ is obtained through the composition of quasi-isometric maps $f_{3}^{-1}$ and $\lambda n . \min f_{2}^{-1}(n)$ applied to $\beta$.

Informally, the proposition above says that any quasiisometry can be decomposed into three parts. The first part is the mapping $f_{1}$ which can be viewed as a "pollution" of $\alpha$, the second part is the map $f_{2}$ that can be called a "collapsing" map, and the third part is $f_{3}$ that can be called "mixing" since it mixes atomic crossing maps. Note that the mixing part is the one that makes things complicated: below we show that mixing functions preserve componentwise reducibility.

Lemma III.10. Suppose $\alpha \leqslant_{C R} \beta$ and $\beta \leqslant_{Q I} \gamma$ via an atomic crossing map $f: \beta \rightarrow \gamma$. Then $\alpha \leqslant_{C R} \gamma$. 
Proof. Let $\alpha=u_{1} u_{2} \ldots$ and $\beta=v_{1} v_{2} \ldots$ be witness partitions. Let $\gamma=w_{1} w_{2} \ldots$ be a partition such that $\left|w_{n}\right|=\left|v_{n}\right|$ for all $n$. We construct a strictly increasing sequence $\left\{n_{k}\right\}_{k \in \mathbb{N}}$ such that $v_{n_{k}} \ldots v_{n_{k+1}-1} \sqsubseteq w_{n_{k}} \ldots w_{n_{k+1}-1}$ for each $k$. Through the proof we identify a finite sequence $u_{n}$ in a partition $\alpha=u_{1} u_{2} \ldots$ and an interval $\left[\left|u_{1} \ldots u_{n-1}\right|,\left|u_{1} \ldots u_{n}\right|-1\right]$ in $\alpha$, and write as " $a \in u_{n}$ " for given $a \in \mathbb{N}$.

We can assume $f$ satisfies the following conditions without loss of generality:

1) $f$ is characterised by $\left\{a_{i}, b_{i}\right\}_{i \in \mathbb{N}}$ such that $C\left(a_{i}\right) \neq$ $C\left(b_{i}\right)$ for all $i \in \mathbb{N}$.

2) For each $i, a_{i} \in u_{i}$ and $b_{i} \in u_{i+1}$.

The following claim is the crucial part of our proof.

Claim. Let $n \in \mathbb{N}$ be given. If $C\left(b_{n}\right) \in C\left(w_{n+i}\right)$ for some $1 \leqslant i \leqslant|\Sigma|$, then there exists $j$ such that $1 \leqslant j \leqslant|\Sigma|(\Sigma+$ 1) such that $v_{n+1} \ldots v_{n+j} \sqsubseteq w_{n+1} \ldots w_{n+j}$ and $c\left(b_{n+j}\right) \in$ $c\left(w_{n+j+i^{\prime}}\right)$ for some $1 \leqslant i^{\prime} \leqslant|\Sigma|$.

Note that for given $n$, there are $n_{1}, n_{2} \in\{n, \ldots, n+|\Sigma|\}$ such that $n_{1}<n_{2}$ and $C\left(b_{n_{1}}\right)=C\left(b_{n_{2}}\right)$ : this is immediate from the pigeonhole principle. This implies that $C\left(b_{n_{1}}\right) \in$ $C\left(w_{n_{2}}\right)$ and $n_{2}-n_{1} \leqslant|\Sigma|-1$. Also for given $n$, let

$$
A=\left\{m \mid m>n \wedge C\left(a_{m}\right) \notin C\left(w_{n+1} \ldots w_{m}\right)\right\} .
$$

Then $|A| \leqslant|\Sigma|-1$, as $m \in A$ only if $C\left(a_{m}\right) \neq C\left(a_{n}\right)$, and for any distinct $m, m^{\prime} \in A$ we have $C\left(a_{m}\right) \neq C\left(a_{m}^{\prime}\right)$.

Now assume $C\left(b_{n}\right) \in C\left(w_{n+i}\right)$ holds for some $1 \leqslant$ $i \leqslant|\Sigma|$. From the facts above, we have $1 \leqslant m \leqslant|\Sigma|^{2}$ such that for all $m^{\prime} \leqslant|\Sigma|$ we have $C\left(b_{n}\right), C\left(a_{n+m+m^{\prime}}\right) \in$ $C\left(w_{n+1} \ldots w_{n+m+m^{\prime}}\right)$. Notice that from this we have $v_{n+1} \ldots v_{n+m+m^{\prime}} \sqsubseteq w_{n+1} \ldots w_{n+m+m^{\prime}}$. Finally, we can find $m^{\prime}$ and $i^{\prime}$ such that $m^{\prime}<m^{\prime}+i^{\prime} \leqslant|\Sigma|$ and $C\left(b_{n+m+m^{\prime}}\right)=$ $C\left(b_{n+m+m^{\prime}+i^{\prime}}\right)$, hence $C\left(b_{n+m+m^{\prime}}\right) \in C\left(w_{n+m+m^{\prime}+i^{\prime}}\right)$, where $1 \leqslant i^{\prime} \leqslant|\Sigma|$. This $m+m^{\prime}$ is the desired $j$.

Find $n$ so that $u_{1} \ldots u_{n} \sqsubseteq w_{1} \ldots w_{n}$ and $C\left(b_{n}\right) \in C\left(w_{n+i}\right)$ for some $1 \leqslant i \leqslant|\Sigma|$, then iterate finding $j$ in the claim above. This is a procedure that gives the desired $\left\{n_{k}\right\}$.

Theorem III.11. $\alpha \leqslant_{Q I} \beta$ implies $\alpha \leqslant_{C R} \beta$.

Proof. Let $f: \alpha \rightarrow \beta$ be a quasi-isometry. Decompose it into $\alpha \stackrel{f_{1}}{\longrightarrow} \gamma_{1} \stackrel{f_{2}}{\longrightarrow} \gamma_{2} \stackrel{f_{3}}{\longrightarrow} \beta$ according to Proposition $\amalg$ III.8 For any monotonic QI map $g: \alpha^{\prime} \rightarrow \beta^{\prime}$ we easily have $\alpha^{\prime} \leqslant_{C R} \beta^{\prime}$ : Indeed, enumerate all elements of an image of $g$ as $\left\{b_{n}\right\}_{n \in \mathbb{N}}$, where $b_{n}<b_{n+1}$ for all $n$, let $u_{n}=g^{-1}\left(b_{n}\right)$ and $v_{n}=$ $\left[b_{n}, b_{n+1}-1\right]$. Then $\alpha^{\prime}=u_{1} u_{2} \ldots$ and $\beta^{\prime}=v_{1} v_{2} \ldots$ are witnessing partitions. Hence we have $\alpha \leqslant_{C R} \gamma_{2}$, and Lemma III.10 completes the proof.

One could attempt to go further, namely say $\alpha$ and $\beta$ are colour-equivalent if they are componentwise reducible to each other via the same witnessing partitions. It is clear that if $\alpha$ and $\beta$ are colour-equivalent, then they are quasi-isometric. Can we show that $\alpha \sim_{Q I} \beta$ implies their colour-equivalence?

Proposition III.12. There are colour-equivalent $\alpha$ and $\beta$ such that no eventual embeddings witness quasi-isometry between $\alpha$ and $\beta$.
Proof. Consider the following coloured metric spaces over the alphabet $\{0,1\}$ :

$$
\alpha=001^{2} 001^{4} \ldots 1^{2 n} 00 \ldots \text { and } \beta=0101^{2} \ldots 01^{n} \ldots
$$

We now prove that no eventual embeddings exist witnessing quasi-isometry between $\alpha$ and $\beta$.

Assume that $f: \alpha \rightarrow \beta$ and $g: \beta \rightarrow \alpha$ are eventual embeddings with witness constants $A_{f}, B_{f}$ and $A_{g}, B_{g}$. Let $i_{n}$ and $i_{n}+1$ be the sequence of consecutive positions in $\alpha$ with colour 0 . Since $f$ is quasi-isometry we have

$$
\left|f\left(i_{n}+1\right)-f\left(i_{n}\right)\right| \leqslant A_{f}\left|i_{n+1}-i_{n}\right|+B_{f}=A_{f}+B_{f} .
$$

There exists a position $i_{n}$ such that $f\left(i_{n}\right)<f\left(i_{n}+1\right)$ and the number of consecutive $1 \mathrm{~s}$ immediately on the right side of $f\left(i_{n}\right)$ is greater that $A_{f}+B_{f}$. Hence, $f\left(i_{n}+1\right)-f\left(i_{n}\right)>$ $A_{f}+B_{f}$. This is a contradiction with quasi-isometry.

Proposition III.13. There are sequences $\alpha$ and $\beta$ such that $\alpha$ and $\beta$ are quasi-isometric via monotonic embeddings but $\alpha$ and $\beta$ are not colour-equivalent.

Proof. Let $\Sigma=\{0,1, *\}$ and define $\alpha, \beta \in \Sigma^{\omega}$ as follows. We first define $\beta$ by:

$$
010 * \quad 0 * 1 * 0 * * * \quad \ldots \quad 0(*)^{n} 1(*)^{n} 0(*)^{2 n+1} \quad \ldots
$$

Thus, the $n^{t h}$-part of $\beta$ is the string $0(*)^{n} 1(*)^{n} 0(*)^{2 n+1}$. We re-write $\beta$ with subscripts on each 0 and 1 as pointers for easier readability:

$$
0_{0} 1_{0} 0_{\frac{1}{2}} * 0_{1} * 1_{1} * 0_{\frac{3}{2}} * * * \ldots 0_{n}(*)^{n} 1_{n}(*)^{n} 0_{\frac{2 n+1}{2}}(*)^{2 n+1} \ldots
$$

Let $\alpha$ be obtained from $\beta$ by omitting the second occurrence of 0 in the $n^{t h}$-substring of $\beta$. So,

$$
\alpha=01 * \quad 0 * 1 * * * * \quad \ldots \quad 0(*)^{n} 1(*)^{n}(*)^{2 n+1} \ldots
$$

So, the $n^{t h}$-substring of $\alpha$ is $0(*)^{n} 1(*)^{n}(*)^{2 n+1}$. As above we re-write $\alpha$ with with subscripts as pointers:

$$
\alpha=0_{0} 1_{0} * 0_{1} * 1_{1} * * * * \ldots 0_{n}(*)^{n} 1_{n}(*)^{3 n+1} \ldots
$$

Constructing a quasi-isometry $f: \alpha \rightarrow \beta$ is straightforward, since eliminating $0_{\underline{2 n+1}}$ for each $n \in \mathbb{N}$ from $\beta$ we have the sequence $\alpha$. We define $g: \beta \rightarrow \alpha$ as a map that sends certain intervals in $\beta$ to intervals in $\alpha$ monotonically and as equally as possible, as follows:

- $g$ maps each sequence $0_{n}(*)^{n} 1_{n}$ in $\beta$ to the sequence $0_{2 n}(*)^{2 n} 1_{2 n}$ in $\alpha$;

- $1_{n}(*)^{n} 0_{\underline{2 n+1}}$ in $\beta$ to $1_{2 n}(*)^{6 n+2} 0_{2 n+1}$ in $\alpha$; and

- $0_{\frac{2 n+1}{2}}(*)^{2 n+1} 0_{n+1}$ in the string $\beta$ to the sequence $0_{2 n+1}{ }^{2}(*)^{2 n+1} 1_{2 n+1}(*)^{6 n+3} 0_{2(n+1)}$ in $\alpha$.

Then $d(i, j) \leqslant d(g(i), g(j)) \leqslant 6 d(i, j)$ and $g$ is a monotonic embedding, thus it is a witness for quasi-isometric embedding.

For non-colour equivalence, assume the contrary and let $\alpha=u_{1} u_{2} \ldots$ and $\beta=v_{1} v_{2} \ldots$ be a witness. We should have only finitely many $u_{i}$ and $v_{i}$ that contain more than one occurrences of 0 and/or 1 , since otherwise we do not have a bound of $\left|u_{i}\right|$ or $\left|v_{i}\right|$. Let $I \in \mathbb{N}$ be a number such that for all $i \geqslant I$ this does not happen. 
Let $p \geqslant I$ and suppose $u_{p}$ contains $1_{n}$. Then $v_{p}$ contains $1_{n+k}$ for some $k \in \mathbb{Z}$, and for all $1_{n^{\prime}}$ and $p^{\prime} \geqslant I$ such that $u_{p^{\prime}}$ contains $1_{n^{\prime}}, v_{p^{\prime}}$ contains $1_{n^{\prime}+k}$ (otherwise some $u_{p^{\prime}}$ or $v_{p^{\prime}}$ contains multiple $1 \mathrm{~s}$ ).

Now suppose $p^{\prime}>p \geqslant I$ and $u_{p}$ and $u_{p^{\prime}}$ contain $1_{n}$ and $1_{n+1}$, respectively. Then from the construction of $\alpha$ there should be exactly one $q \in\left[p+1, p^{\prime}-1\right]$ with the corresponding interval $u_{q}$ contains 0 . On the other hand $v_{p}$ and $v_{p^{\prime}}$ contain $1_{n+k}$ and $1_{n+k+1}$, respectively, and from the construction of $\beta$ we should have two elements in $\left[p+1, p^{\prime}-1\right]$ with the corresponding interval contains 0 , which is the contradiction.

\section{BÜCHI AUTOMATA AND LARGE SCALE GEOMETRIES}

Let $L \subseteq \Sigma^{\omega}$ be a language, where we assume that $\Sigma=$ $\{0,1\}$. The notion of quasi-isometry leads us to consider the quasi-isometry types of strings from $L$ :

Definition IV.1. An atlas is a set of quasi-isometry types. In particular, the atlas defined by the language $L$ is the set $[L]=\{[\alpha] \mid \alpha \in L\}$, where $[\alpha]$ is the quasi-isometry type of $\alpha$.

A question of a general character is concerned with understanding the set $[L]$ given a description of $L$. In particular, a natural question is if one can decide that $\left[L_{1}\right]=\left[L_{2}\right]$ given descriptions of languages $L_{1}$ and $L_{2}$. In this section, we study the atlases defined by Büchi automata recognisable languages. We show that for Büchi automata recognisable languages $L_{1}$ and $L_{2}$ there is an efficient algorithm that decides if $\left[L_{1}\right]=\left[L_{2}\right]$. We recall basic definitions for Büchi automata.

Definition IV.2. A Büchi automaton $\mathcal{M}$ is a quadruple $(S, \iota, \Delta, F)$, where $S$ is a finite set of states, $\iota \in S$ is the initial state, $\Delta \subset S \times \Sigma \times S$ is the transition table, and $F \subseteq S$ is the set of accepting states.

A run of $\mathcal{M}$ on $\alpha=\sigma_{0} \sigma_{1} \cdots \in \Sigma^{\omega}$ is a sequence of states $r=s_{0}, s_{1}, \ldots$ such that $s_{0}=\iota$ and $\left(s_{i}, \sigma_{i}, s_{i+1}\right) \in \Delta$ for all $i \in \omega$. The run is accepting if the set $\operatorname{Inf}(r)=\left\{s: \exists^{\infty} i\left(q_{i}=\right.\right.$ $s)$ \} has a state from $F$. The automaton $\mathcal{M}$ accepts the string $\alpha$ if it has an accepting run on it. The language accepted by $\mathcal{M}$, denoted $L(\mathcal{M})$, is the set of all infinite words accepted by $\mathcal{M}$.

Let $\mathcal{M}$ be a Büchi automaton. Our goal is to describe the atlas defined by the language $L(\mathcal{M})$. We call such $[L]$ Büchi recognisable. We need to do some state space analysis of the automation $\mathcal{M}$.

A loop (in $\mathcal{M}$ ) is a path $L$ of states $s_{1}, \ldots, s_{n+1}$ in the state space $S$ such that $s_{1}=s_{n+1}$. We say that a word $\sigma_{1} \ldots \sigma_{n}$ labels the loop $L$ if $\left(s_{i}, \sigma, s_{i+1}\right) \in \Delta$ for $1 \leqslant i \leqslant n$. If all symbols $\sigma_{i}$ are 0 only (or 1 only), then we say that the loop is 0 -loop (or respectively, 1-loop). In the loop $L$ we do not require that the states are pairwise distinct. Any state in a loop is a loop state.

The state space $S$ can naturally be considered as a directed graph, where the edges between states are transitions of $\mathcal{M}$ with labels removed. Hence, we can write $S$ as a disjoint union of its strongly connected components $(\operatorname{sccs})$. Call a scc $X \subseteq S$ non-trivial if $|X|>1$, where $|X|$ is the cardinality of $X$. Thus we write $S$ as the union $T \cup S_{1} \cup \ldots \cup S_{k}$, where $T$ is the union of trivial strongly connected components and each $S_{i}$ is a nontrivial scc. Every successful run of $\mathcal{M}$ on every infinite string $\alpha$ eventually ends up in one of the $\operatorname{sccs} S_{i}$. Consider Büchi automata $\mathcal{M}_{1}, \ldots, \mathcal{M}_{k}$ such that the initial states and the state diagrams of $\mathcal{M}_{i}$ all coincide with that of $\mathcal{M}$ but $F_{i}=F \cap S_{i}$. It is clear that

$$
L(\mathcal{M})=L\left(\mathcal{M}_{1}\right) \cup \ldots \cup L\left(\mathcal{M}_{k}\right) .
$$

Hence describing the atlas $[L(\mathcal{M})]$ boils down to describing the atlases of $\left[L\left(\mathcal{M}_{i}\right)\right], i=1, \ldots, k$. Assume that $\alpha$ is accepted by $\mathcal{M}_{i}$. Then we can write $\alpha$ as $v \alpha^{\prime}$ such that an accepting run of $\mathcal{M}_{i}$ after reading $v$ stays inside $S_{i}$. Therefore, the large scale geometry $[\alpha]$ of $\alpha$ is quasi-isometric to either $\left[0 \alpha^{\prime}\right]$ or $\left[1 \alpha^{\prime}\right]$ (or both). Therefore, we can postulate the following assumption till the end of this section unless otherwise stated.

Postulate. The state space $S$ can be written as $\left\{q_{0}\right\} \cup S^{\prime}$ such that (1) $q_{0}$ is the only initial state and $q_{0} \notin S^{\prime}$, (2) $S^{\prime}$ is the only nontrivial strongly connected component of $\mathcal{M}$, and (3) any transition from $q_{0}$ goes into $S^{\prime}$.

Lemma IV.3. If $\mathcal{M}$ has a 0-loop and a 1-loop, then the atlas $[L(\mathcal{M})]$ coincides with the atlas $\left[\Sigma^{\omega}\right] \backslash X$ for some $X \subseteq$ $\left\{\left[0^{\omega}\right],\left[1^{\omega}\right],\left[10^{\omega}\right],\left[01^{\omega}\right]\right\}$.

Proof. Assume that $L_{0}$ is a 0-loop and $L_{1}$ is a 1-loop. Let $\alpha$ be a string with $\omega$-many 0 s and $\omega$-many 1 s. Say, $\alpha=0^{n_{0}} 1^{m_{0}} 0^{n_{1}} 1^{m_{1}} \ldots$ We can build a $\beta$ of the form

$\begin{array}{lllllll}u & 0^{n_{1}^{\prime}} v 1^{m_{1}^{\prime}} w & 0^{n_{2}^{\prime}} v 1^{m_{2}^{\prime}} w & 0^{n_{3}^{\prime}} v 1^{m_{3}^{\prime}} w & \ldots & 0^{n_{i}^{\prime}} v 1^{m_{i}^{\prime}} w & \ldots\end{array}$

such that (1) $\beta$ is accepted by $\mathcal{M}$, (2) $n_{i}=n_{i}^{\prime}+c_{i}$ and $m_{i}=m_{i}^{\prime}+c_{i}^{\prime}$, where $c_{i}<|S|$ and $c_{i}^{\prime}<|S|$ for all $i$, (3) $v$ is a string that labels a path from a state $s_{0}$ in $L_{0}$ to a state $s_{1}$ in $L_{1}$, and (4) $w$ is a string that labels a path from $s_{1}$ to $s_{0}$, and (5) $v$ is a string that labels a path from the initial state to $s_{0}$. This implies that $\alpha$ and $\beta$ are colour-equivalent. Hence they are quasi-isometric.

Note that none of the strings $0^{\omega}, 1^{\omega}, 10^{\omega}, 01^{\omega}$ is quasiisometric to a string with both $\omega$-many 0 s and $1 \mathrm{~s}$. Also, these four strings are pairwise not quasi-isometric. Hence, the choice of $X$ is dependent on whether or not $\mathcal{M}$ accepts some (or all) of these four strings. For instance, $0^{\omega} \in X$ iff $0^{\omega}$ is not accepted by $\mathcal{M}$.

Lemma IV.4. If $\mathcal{M}$ has no 0-loop and has no 1-loop, then the atlas $[L(\mathcal{M})]$ equals the singleton atlas $\left[\left\{(01)^{\omega}\right\}\right]$.

Proof. Let $\alpha=\sigma_{0} \sigma_{1} \ldots$ be a string accepted by $\mathcal{M}$. Let $\rho=$ $s_{1}, s_{2}, \ldots$ be an accepting run of $\mathcal{M}$ on $\alpha$. We can write $\alpha$ as $w_{1} w_{2} \ldots$ so that (1) the length of each $w_{i}$ is bounded by $|S|$ and (2) the run $\rho$ along each $w_{i}$ has a loop; that loop within $w_{i}$ contains both 0 and 1 . This implies that $(01)^{\omega}$ and $\alpha$ are colour-equivalent. Hence they are quasi-isometric. 
Lemma IV.5. Suppose that $\mathcal{M}$ contains no 1-loop but has a 0 -loop $L_{0}$. Then the atlas $[L(\mathcal{M})]$ coincides with one of the following atlases:

$$
\begin{gathered}
{\left[\left\{0^{\omega}\right\}\right],\left[\left\{10^{\omega}\right\}\right],\left[\left\{0^{\omega}, 10^{\omega}\right\}\right],} \\
{\left[\left\{0^{n_{1}} 10^{n_{2}} 10^{n_{3}} \ldots \mid n_{i}>0\right\} \backslash\left\{0^{\omega}\right\}\right] \text { or }} \\
{\left[\left\{0^{n_{1}} 10^{n_{2}} 10^{n_{3}} \ldots \mid n_{i}>0\right\} \cup\left\{0^{\omega}\right\}\right] .}
\end{gathered}
$$

Proof. The assumption of the lemma implies that if $\alpha$ is accepted by $\mathcal{M}$ then (1) $\alpha$ contains infinitely many 0 s and (2) the lengths of any sequence of consecutive 1s occurring in $\alpha$ is bounded by $|S|$. If $L(\mathcal{M})=V 0^{\omega}$ for some regular language $V \subseteq \Sigma^{\star}$, then the atlas $[L(\mathcal{M})]$ is either $\left[\left\{0^{\omega}\right\}\right]$ or $\left[\left\{10^{\omega}\right\}\right]$ or $\left[\left\{0^{\omega}, 10^{\omega}\right\}\right]$. So, we assume that $\mathcal{M}$ accepts a string with $\omega$ many 1s'. Let $\alpha$ be any string of the form $0^{t_{1}} 10^{t_{2}} 1 \ldots$, where all $t_{i}>0$ for all $i$. We can build a string $\beta$ of the form $\begin{array}{llllllll}u & 0^{n_{1}} v & 0^{n_{2}} v & 0^{n_{3}} v & \ldots & 0^{n_{i}} v & \ldots \text { such that }(1) \beta \text { is accepted }\end{array}$ by $\mathcal{M}$, (2) $t_{i}=n_{i}+c_{i}$, where $c_{i}<|S|$ for all $i$, and (3) $v$ is a string that labels a path from a state $s_{0}$ in $L_{0}$ back to $s_{0}$, and $v$ contains 1 . The string $\beta$ is quasi-isometric to string $\beta^{\prime}$ obtained from $\beta$ by replacing all occurrences of $v$ with 1 and removing the prefix $u$. In turn $\beta^{\prime}$ is colour equivalent to $\alpha$. Hence, $\alpha \sim_{Q I} \beta$.

Now suppose that $\beta$ is accepted by $\mathcal{M}$ and $\beta$ has infinitely many $1 \mathrm{~s}$. Then $\beta$ is of the form $u 0^{n_{1}} 1^{m_{1}} 0^{n_{2}} 1^{m_{2}} \ldots$ where we have $n_{i}, m_{i}>0$ for all $i$. As noted above the numbers $m_{i}$ are uniformly bounded. The string $\beta$ is quasi-isometric to string $\beta^{\prime}$ obtained from $\beta$ by replacing all $1^{m_{i}}$ with just 1 and removing the prefix $u$. Clearly $\beta^{\prime}$ is of the form desired. Note that $\mathcal{M}$ accepts the string quasi-isometric to $10^{\omega}$. Hence, if $0^{\omega}$ is accepted by $\mathcal{M}$ then the atlas $[L(\mathcal{M})]$ coincides with $\left[\left\{0_{1}^{n} 10^{n_{2}} 10^{n_{3}} \ldots \mid n_{i}>0\right\} \cup\left\{0^{\omega}\right\}\right]$. Otherwise, the atlas $[L(\mathcal{M})]$ equals $\left[\left\{0_{1}^{n} 10^{n_{2}} 10^{n_{3}} \ldots \mid n_{i}>0\right\} \backslash\left\{0^{\omega}\right\}\right]$.

Lemma IV.6. Suppose that $\mathcal{M}$ contains no 0-loop but has a 1-loop $L_{0}$. Then the atlas $[L(\mathcal{M})]$ coincides with one of the following atlases:

$$
\begin{gathered}
{\left[\left\{1^{\omega}\right\}\right],\left[\left\{01^{\omega}\right\}\right],\left[\left\{1^{\omega}, 01^{\omega}\right\}\right],} \\
{\left[\left\{1^{n_{1}} 01^{n_{2}} 01^{n_{3}} \ldots \mid n_{i}>0\right\} \backslash\left\{1^{\omega}\right\}\right] \text { or }} \\
{\left[\left\{1^{n_{1}} 01^{n_{2}} 01^{n_{3}} \ldots \mid n_{i}>0\right\} \cup\left\{1^{\omega}\right\}\right] .}
\end{gathered}
$$

We now remove our postulate put on the state space of Büchi automata. Using equality $(\star)$ given above, and the lemmas, we obtain the following characterisation result:

Theorem IV.7. Any Büchi recognisable atlas $[L]$ is a union from the following list of atlases:

- $\left[\Sigma^{\omega}\right] \backslash X$, where $X \subseteq\left\{\left[0^{\omega}\right],\left[1^{\omega}\right],\left[10^{\omega}\right],\left[01^{\omega}\right]\right\}$.

- $\left[\left\{0^{n_{1}} 10^{n_{2}} 10^{n_{3}} \ldots \mid n_{i}>0\right\} \backslash\left\{0^{\omega}\right\}\right], \quad\left[\left\{1^{\omega}\right\}\right]$,

- $\left[\left\{0^{n_{1}} 10^{n_{2}} 10^{n_{3}} \ldots \mid n_{i}>0\right\} \cup\left\{0^{\omega}\right\}\right], \quad\left[\left\{0^{\omega}\right\}\right]$,

- $\left[\left\{01^{\omega}\right\}\right],\left[\left\{10^{\omega}\right\}\right],\left[\left\{0^{\omega}, 10^{\omega}\right\}\right],\left[\left\{1^{\omega}, 01^{\omega}\right\}\right]$,

- $\left[\left\{1^{n_{1}} 01^{n_{2}} 01^{n_{3}} \ldots \mid n_{i}>0\right\} \backslash\left\{1^{\omega}\right\}\right]$,

- $\left[\left\{1^{n_{1}} 01^{n_{2}} 01^{n_{3}} \ldots \mid n_{i}>0\right\} \cup\left\{1^{\omega}\right\}\right]$.

We obtain the following complexity-theoretic result solving the equality problem for Büchi recognisable atlases. In comparison, the equality problem for Büchi automata is PSPACE complete.
Corollary IV.8. There exists an algorithm that, given Büchi automata $\mathcal{A}$ and $\mathcal{B}$, decides if the atlases $[L(\mathcal{A})]$ and $[L(\mathcal{B})]$ coincide. Furthermore, the algorithm runs in linear time on the size of the input automata.

Proof. Let $\mathcal{M}$ be a Büchi automaton. Our goal is to find atlases from the list provided in Theorem IV.7 such that the union of these atlases coincides with the atlas $[L(\mathcal{M})]$. For this, we decompose $\mathcal{M}$ into $k$ automata $\mathcal{M}_{1}, \ldots, \mathcal{M}_{k}$ so that the equality $(\star)$ holds and each $\mathcal{M}_{i}$ satisfies the postulate. This decomposition can be done in linear time on the size of $\mathcal{M}$ (e.g. using Tarjan's algorithm that decomposes a directed graph into strongly connected components). Now for each $\mathcal{M}_{i}$, we check the assumptions of Lemma IV.3 through Lemma IV.6. This can also be done in linear time.

As an example, the assumptions of Lemma IV.3 can be checked as follows. Assume that the automaton under consideration is $\mathcal{M}_{i}$. In order to check if $\mathcal{M}_{i}$ contains a 0 -loop, we remove all transitions labeled with 1 from the state diagram of $\mathcal{M}_{i}$. This gives us a directed graph whose edges are transitions labelled with 0. In this graph we check if there is a loop. If there exists a loop, then $\mathcal{M}_{i}$ has a 0-loop. Otherwise $\mathcal{M}_{i}$ does not have a 0 -loop. Similarly, to check if $\mathcal{M}_{i}$ contains a 1-loop, we remove all transitions labeled with 1 . This gives us a directed graph. If there is a loop in this directed graph, then $\mathcal{M}_{i}$ has a 1-loop; Otherwise, not. To find the set $X$ from (the statement of) the Lemma we just need to check which of the strings $0^{\omega}, 1^{\omega}, 10^{\omega}, 01^{\omega}$ is accepted by $\mathcal{M}_{i}$. This can also be done in linear time on the size of $\mathcal{M}_{i}$.

The argument shows that we can find, in linear time on size of $\mathcal{M}$, the atlases from the list provided in Theorem IV.7 such that the union of these atlases is the atlas $[L(\mathcal{M})]$. Thus, given Büchi automata $\mathcal{A}$ and $\mathcal{B}$ we can decide in linear time if $[L(\mathcal{A})]=[L(\mathcal{B})]$.

\section{THE QUASI-ISOMETRY PROBLEM}

The quasi-isometry problem $Q I P$ is to find if, given strings $\alpha$ and $\beta$, there is a quasi-isometry from $\alpha$ to $\beta$ :

$$
Q I P=\left\{(\alpha, \beta) \mid \alpha, \beta \in \Sigma^{\omega} \&[\alpha] \leqslant_{Q I}[\beta]\right\} .
$$

Let $\alpha$ and $\beta$ be coloured metric spaces and $A, B$ be constants. Our goal is to construct a rooted tree $T(\alpha, \beta, A, B)$ such that the following properties hold:

1 . The tree $T(\alpha, \beta, A, B)$ is finitely branching and computable in $\alpha$ and $\beta$. In particular, if $\alpha$ and $\beta$ are computable then so is $T(\alpha, \beta, A, B)$.

2 . For any $n$ one can effectively compute, with an oracle for $\alpha$ and $\beta$, the number of nodes in the tree at distance $n$ from the root.

3. The tree $T(\alpha, \beta, A, B)$ is infinite iff there exists an $(A, B)$ quasi-isometric map from $\alpha$ into $\beta$.

4. There is a bijection between $(A, B)$-quasi-isometric maps from $\alpha$ to $\beta$ and the set $[T(\alpha, \beta, A, B)]$ of all infinite paths of the tree $T(\alpha, \beta, A, B)$.

Informally, the nodes of $T(\alpha, \beta, A, B)$ are finite partial functions that can potentially be extended to $(A, B)$-quasiisometric maps from $\alpha$ to $\beta$. Formally: 
1. The root of the tree $T(\alpha, \beta, A, B)$ is the empty set $\emptyset$, that is, nowhere defined partial function.

2. Let $x$ be a node of the tree $T(\alpha, \beta, A, B)$ constructed so far, and $f_{x}$ be the partial function associated with $x$. We identify $x$ and $f_{x}$. We assume that

1) $x$ is at distance $n$ from the root, and $f_{x}=$ $\left\{\left(0, i_{0}\right), \ldots,\left(n-1, i_{n-1}\right)\right\}$.

2) For all $k, m$ from the domain of $f_{x}$ we have that $f_{x}$ is colour preserving and the $(A, B)$-quasi-isometry condition is satisfied:

$$
\begin{gathered}
(1 / A) \cdot d(k, m)-B<d\left(f_{x}(k), f_{x}(m)\right) \\
<A \cdot d(k, m)+B .
\end{gathered}
$$

We list all the extension $\phi$ of $f_{x}$ such that the domain of $\phi$ is the set $\{0,1, \ldots, n\}$ and $\phi$ satisfies the the $(A, B)$ quasi-isometry condition. The children of $f_{x}$ will be all these functions $\phi$ extending $f_{x}$.

In Part 2 of the construction the number of extensions $\phi$ of $f_{x}$ is finite since these extensions must satisfy the quasiisometry condition. Moreover, the number of these extensions is computed with an oracle for $\alpha$ and $\beta$. If such extensions $\phi$ of $f_{x}$ do not exist, then $x$ is a leaf.

Lemma V.1. The tree $T(\alpha, \beta, A, B)$ is infinite iff there is an $(A, B)$-quasi-isometric map from $\alpha$ into $\beta$. There is a bijection between all infinite paths of $T(\alpha, \beta, A, B)$ and $(A, B)$-quasiisometric maps from $\alpha$ to $\beta$.

Proof. If $g: \alpha \rightarrow \beta$ is an $(A, B)$-quasi-isometric map from $\alpha$ to $\beta$ then the sequence $\left\{g_{i}\right\}_{i \in \omega}$, where $g_{i}$ is the restriction of $g$ on the initial segment $\{0, \ldots, i\}$, is an infinite path through the tree $T(\alpha, \beta, A, B)$. If $x_{1}, x_{2}, \ldots$ is an infinite path through the tree $T(\alpha, \beta, A, B)$ then $f$ defined as the limit of the sequence $f_{x_{1}}, f_{x_{2}}, \ldots$ is an $(A, B)$-quasi-isometry from $\alpha$ to $\beta$.

The next theorem that solves the quasi-isometry problem.

\section{Theorem V.2. The following statements are true:}

1) Given quasi-isometric strings $\alpha$ and $\beta$, there exists $a$ quasi-isometry between $\alpha$ and $\beta$ computable in the halting set relative to $\alpha$ and $\beta$.

2) The quasi-isometry problem between computable strings is a complete $\Sigma_{3}^{0}$-set.

Proof. Assume that $\alpha$ and $\beta$ are $(A, B)$-quasi-isometric. The tree $T(\alpha, \beta, A, B)$ is infinite by the Lemma above. Such a tree has a path computable in the halting set for the tree [10]. The tree, as we constructed above, is computable in $\alpha$ and $\beta$. This proves the first part.

We prove the second part of the theorem. By Lemma V.1 computable strings $\alpha$ and $\beta$ are quasi-isometric if and only if there exists are constants $A, B$ such that the computable tree $T(\alpha, \beta, A, B)$ is infinite. This is a $\Sigma_{3}^{0}$-statement. Hence, the quasi-isometry problem between computable strings $\alpha$ and $\beta$ is a $\Sigma_{3}^{0}$-set. To prove that the problem is complete, we reduce a $\Sigma_{3}^{0}$-complete problem to the quasi-isometry problem. Below is an informal explanation of the reduction.
Let $W_{0}, W_{1}, \ldots$ be a standard enumeration of all c.e. sets. It is known that the set Fin $=\left\{i \mid W_{i}\right.$ is finite $\}$ is a $\Sigma_{3^{-}}^{0}$ complete problem [12]. For each $W_{i}$ we need to construct $\alpha_{i}$ and $\beta_{i}$ two infinite strings such that $W_{i}$ is finite if and only if $\alpha_{i}$ and $\beta_{i}$ are quasi-isometric. For this we start enumerating $W_{i}$ by stages $1,2, \ldots$ At stage 0 , we consider $\alpha_{i, 0}$ and $\beta_{i, 0}$ finite prefixes that can be extended to a $(1,1)$-quasi-isometry and set $A_{0}=B_{0}=1$. By stage $s$ we will have a finite prefixes $\alpha_{i, s-1}$ of $\alpha_{i}$ and $\beta_{i, s-1}$ of $\beta_{i}$ built. If at stage $s$ the enumeration of $W_{i}$ outputs a new element, then we start extending $\alpha_{s}$ and $\beta_{s}$ so that the following holds. If $W_{i}$ never increases its size from stage $s$ on then $\alpha$ and $\beta$ are $\left(A_{s}, B_{s}\right)$ quasi-isometric but not $\left(A_{s-1}, B_{s-1}\right)$-quasi-isometric, where $A_{s}>A_{s-1}$ and $B_{s}>B_{s-1}$. This can easily be achieved in two steps. In the first step, one ensures that a large part of $\alpha$ contains a long consecutive sequence of elements of one colour, and the same positions of $\beta$ have another colour. This will guarantee that $\alpha_{i}$ and $\beta_{i}$ are not $\left(A_{s-1}, B_{s-1}\right)$-quasiisometric. In the second step, one ensures that those long sequences in the first step do not conflict with $\left(A_{s}, B_{s}\right)$-quasiisometry and one can continue on extending $\alpha_{i, s}$ and $\beta_{i, s}$ so that they are $\left(A_{s}, B_{s}\right)$-quasi-isometric. Thus, if $W_{i}$ is infinite then there is an infinite sequence $s_{1}, s_{2}, \ldots$ of increasing stages at which the enumeration of $W_{i}$ increases the size of $W_{i}$. Hence, from stage $s_{i}$ on $\alpha$ and $\beta$ are not $\left(A_{s_{i}}, B_{s_{i}}\right)$-quasiisometric. This implies that $W_{i}$ is infinite then $\alpha_{i}$ and $\beta_{i}$ are not quasi-isometric.

\section{ASYMPTOTIC CONES}

Let $\mathcal{F}$ be a non-principal ultra-filter on $\omega$. Recall that a non-principal ultra-filter is a non-empty maximal subset of $P(\omega)$ that satisfies the following properties: (1) No finite set belongs to $\mathcal{F}$. So, $\mathcal{F}$ does not contain the empty set; (2) For all $A, B \in \mathcal{F}$ we have $A \cap B \in \mathcal{F}$; (3) For all $A, B \subseteq \omega$ if $A \in \mathcal{F}$ and $A \subseteq B$ then $B \in \mathcal{F}$.

Every ultrafilter $\mathcal{F}$ has the following two properties: (1) For every set $A \subseteq \omega$, either $A \in \mathcal{F}$ or $\omega \backslash A \in \mathcal{F}$. (2) For all pairwise disjoint sets $A, B \subseteq \omega$ if $A \cup B \in \mathcal{F}$ then either $A \in \mathcal{F}$ or $B \in \mathcal{F}$.

Let $\alpha \in \Sigma^{\omega}$ and $s$ be a strictly increasing monotonic function on $\omega$ with $s(0)=1$. Call the mapping $s(n)$ a scaling factor. Define the following sequence of metric spaces:

$$
X_{0, \alpha}=\left(\alpha, d_{0}\right), X_{1, \alpha}=\left(\alpha, d_{1}\right), \ldots, X_{n, \alpha}=\left(\alpha, d_{n}\right), \ldots
$$

where $d_{n}(i, j)=|i-j| / s(n)$. Informally, we move $\alpha$ away from us by scaling the metric down. For instance, in the metric space $X_{0, \alpha}$ the distance from 0 to $s(n)$ equals $s(n)$, while in $X_{n, \alpha}$ the distance from 0 to $s(n)$ is 1 . We assume that the domains of these metric spaces are disjoint pairwise: $X_{i, \alpha} \cap$ $X_{j, \alpha}=\emptyset$ for all $i \neq j$.

Let $\mathbf{a}=\left(a_{n}\right)_{n \geqslant 0}$ be a sequence, where each $a_{n} \in X_{n, \alpha}$ for all $n$. Call the sequence bounded if there is a constant $L$ such that the set $\left\{n \mid d_{n}\left(0, a_{n}\right)<L\right\} \in \mathcal{F}$. Let $\mathbf{B}(\mathcal{F}, s)$ be the set of all bounded sequences. Say that two bounded sequences a and $\mathbf{b}$ are $\mathcal{F}$-equivalent, written $\mathbf{a} \sim_{\mathcal{F}} \mathbf{c}$, if $\left\{n \mid d_{n}\left(a_{n}, b_{n}\right) \leqslant\right.$ $\epsilon\} \in \mathcal{F}$ for every $\epsilon$. 
Definition VI.1. Define the asymptotic cone of $\alpha$, written Cone $(\alpha, \mathcal{F}, s)$, with respect to the scaling function $s(n)$ and the ultra-filter $\mathcal{F}$ to be the factor set

$$
\mathbf{B}(\mathcal{F}, s) / \sim_{\mathcal{F}}
$$

equipped with the following metric $D$ and colour $C$ :

1) $D(\mathbf{a}, \mathbf{b})=r$ iff $\left\{n \mid r-\epsilon \leqslant d_{n}\left(a_{n}, b_{n}\right) \leqslant r+\epsilon\right\} \in \mathcal{F}$ for all $\epsilon>0$.

2) $C(\mathbf{a})=\sigma$ iff $\left\{n \mid a_{n}\right.$ has colour $\left.\sigma\right\} \in \mathcal{F}$.

This definition implies that some elements a of the asymptotic cone might have several colours.

Lemma VI.2. The set $\{\mathbf{a} \in \operatorname{Cone}(\alpha, \mathcal{F}, s) \mid \sigma \in C(\mathbf{a})\}$ is closed for each $\sigma \in \Sigma$,

Proof. Let $\mathbf{a}$ be a limit point of the set above, $\mathbf{a}_{\mathbf{n}}=$ $\left(a_{n m}\right)_{m \geqslant 0} \in \operatorname{Cone}(\alpha, \mathcal{F}, s)$ be a point of colour $\sigma$ such that $D\left(\mathbf{a}, \mathbf{a}_{\mathbf{n}}\right) \leqslant 2^{-n}$. Also let $r=D(\mathbf{0}, \mathbf{a}), r_{n}=D\left(\mathbf{0}, \mathbf{a}_{\mathbf{n}}\right)$, and $i_{m}$ be a number such that $i_{m} \leqslant m$ and $\left|r-d_{n}\left(0, a_{i_{m} m}\right)\right| \leqslant$ $\left|r-d_{n}\left(0, a_{j m}\right)\right|$ for all $j \leqslant m$. Then $\mathbf{b}=\left(a_{i_{n} n}\right)_{n \geqslant 0} \sim \mathbf{a}$ and $\sigma \in C(\mathbf{b})$ : indeed, let $A_{n}=\left\{m \in \mathbb{N}|| r_{n}-d_{m}\left(0, a_{n m}\right) \mid \leqslant\right.$ $\left.2^{-n}\right\}$. Then for given $n$,

$$
\begin{aligned}
& \left\{m \in \mathbb{N}|| r-d_{m}\left(0, b_{m}\right) \mid \leqslant 2^{-(n+1)}\right\} \\
\supseteq & \left\{m \in \mathbb{N}|| r-d_{m}\left(0, a_{i_{m} m}\right) \mid \leqslant 2^{-(n+1)}\right\} \cup[n, \infty) \\
\supseteq & A_{n+2} \cup[n, \infty) \in \mathcal{F} .
\end{aligned}
$$

Also $\left\{m \in \mathbb{N}|| r-d_{m}\left(0, a_{m}\right) \mid \leqslant 2^{-(n+1)}\right\} \in \mathcal{F}$, hence

$$
\left\{m \in \mathbb{N} \mid d_{m}\left(a_{m}, b_{m}\right) \leqslant 2^{-n}\right\} \in \mathcal{F} .
$$

Theorem VI.3. For eventually periodic word $\alpha=u v^{\omega}$ all asymptotic cones Cone $(\alpha, \mathcal{F}, s)$ equal the coloured metric space $\left(\mathbb{R}_{\geqslant 0} ; d, C\right)$ such that every real $r>0$ has colours of $v$ and the real 0 has colours of both $u$ and $v$.

Proof. Let $\mathcal{F}$ be any filter and $s: \omega \rightarrow \omega$ be a monotonic function. It is easy to see that 0 has all colours of both $u$ and $v$. Let $r \in \mathbb{R}$ and $r>0$. For any $\sigma$ present in $v$ there is a sequence $\left(a_{n}\right)_{n \geqslant 0}$ such that $a_{n} \in X_{n, \alpha}$, the colours of $a_{n}$ all are $\sigma$ and for every $\epsilon>0$ there is an $N_{\epsilon}$ such that for all $n>N_{\epsilon}$ we have $\left|d_{n}\left(a_{n}, 0\right)-r\right| \leqslant \epsilon$. Indeed, for each $n \in \mathbb{N}$ such that $|u| / s\left(N_{\epsilon}\right)<r$ let $a_{n}$ be any element which has the colour $\sigma$ and satisfies $(|u|+i|v|) / s(n) \leqslant d_{n}\left(0, a_{n}\right)<(|u|+$ $(i+1)|v|) / s(n)$, where $i$ is the unique integer which satisfies $(|u|+i|v|) / s(n) \leqslant r<(|u|+(i+1)|v|) / s(n) . \mid d_{n}\left(a_{n}, 0\right)-$ $r|\leqslant| v \mid / s(n)$. This inequality implies the claim.

We now prove a theorem akin to a known result in geometric group theory stating that there are non-quasi-isometric groups that realise the same cones. For instance, in [2] [3] it is proved that all asymptotic cones of non-elementary hyperbolic group are isometric.

Theorem VI.4. There are non-quasi-isometric strings $\alpha, \beta \in$ $\{0,1\}^{\omega}$, a scale factor $s(n)$, and filter $\mathcal{F}$ such that Cone $(\alpha, \mathcal{F}, s)=\operatorname{Cone}(\beta, \mathcal{F}, s)$ and $\alpha, \beta \in \mathcal{X}(1)$.

Proof. Our scale factor is $s(n)=2^{n}$. The string $\alpha$ is such that $\alpha(i)=1$ if and only if $i$ is a power of 2 . Let $\mathcal{F}$ be any ultra-filter. Consider the cone $\operatorname{Cone}(\alpha, \mathcal{F}, s)$. It is note hard to see that the cone coincides with the coloured metric space $\left(\mathbb{R}_{\geqslant 0} ; d, C\right)$, where $d$ is the usual metric on reals, all reals have colour 0 , and a real $r$ has also colour 1 iff $r$ is an integer power of 2 or $r=0$.

So, we need to construct $\beta$ and a filter $\mathcal{F}$ such that $\alpha$ and $\beta$ are not quasi-isometric but the cone $\operatorname{Cone}(\beta, \mathcal{F}, s)$ coincides with the above coloured metric space $\left(\mathbb{R}_{\geqslant 0} ; d, C\right)$. Let $\beta$ be a string of the form

$$
0^{m_{0}} 10^{m_{1}} 10^{m_{2}} 10^{m_{3}} \ldots 0^{m_{k}} 10^{m_{k+1}} \ldots
$$

such that the following properties hold:

1) The positions where $\beta$ contains 1 are powers of two, and let us list these positions as the sequence $2^{n_{0}}, 2^{n_{1}}, 2^{n_{2}}, \ldots$

2) For each $i \in \omega$ we have $2 n_{i}<n_{i+1}$.

3) The strings $\alpha$ and $\beta$ are not quasi-isometric.

Now we construct our filter $\mathcal{F}$. Let $X$ be the set

$$
\left\{n_{0}, n_{0}+1, \ldots, 2 n_{0}, \ldots, n_{k}, n_{k}+1, \ldots, 2 n_{k}, \ldots\right\} .
$$

Call the sequences of the form $n_{k}, n_{k}+1, \ldots, 2 n_{k}$ blocks of the set $X$. Note that the lengths of the blocks is unbounded. For each integer $i$, consider the set $X+i=\{x+i \mid x \in$ $X, x+i \geqslant 0\}$. Since the sizes of the blocks is unbounded, the collections of sets is a bases of a filter:

$$
X, X+1, X-1, X+2, X-2, \ldots, X+i, X-i, \ldots
$$

Let $\mathcal{F}$ be the ultrafilter that contains the collection.

Consider the cone $\operatorname{Cone}(\beta, \mathcal{F}, s)$. We can view the domain of this cone as the set $\mathbb{R}_{\geqslant 0}$. It is not too hard to note that every real $r$ gets colour 0 in the cone $\operatorname{Cone}(\beta, \mathcal{F}, s)$. Now we need to show that $r$ gets colour 1 if and only if $r$ is an integer power of 2. It suffices to show that if $r$ is an integer power of 2 then $r$ has colour 1. But, this is implied by the fact that the sets $X+i$ are in $\mathcal{F}$. Indeed, assume that $r$ is of the form $2^{i}$. Then, since $X+i \in \mathcal{F}$, from the definition of $\operatorname{Cone}(\beta, \mathcal{F}, s)$ we see that $r$ has colour 1 .

To finish the proof, we just need to show that we can select the sequence $n_{0}, n_{1}, \ldots$ such that $\alpha$ and $\beta$ are not quasiisometric. To see this, let $n_{k+1}=2 n_{k}+2$ : then we can show that $\alpha$ and $\beta$ are not quasi-isometric in the same manner as the proof of Theorem II.5

In contrast to the theorem above, we show that the same coloured metric space can produce two asymptotic cones that are not quasi-isometric. This is similar to the result in geometric group theory where one group can realise two non homeomorphic asymptotic cones [1].

Theorem VI.5. There is a sequence $\alpha$, scaling factors $s_{0}$ and $s_{1}$ so that for all ultrafilters $\mathcal{F}$ cones $\operatorname{Cone}\left(\alpha, \mathcal{F}, s_{0}\right)$ and Cone $\left(\alpha, \mathcal{F}, s_{1}\right)$ are not quasi-isometric.

Proof. We construct $\alpha$ and two scaling factors $s_{0}$ and $s_{1}$ such that in the asymptotic cone $\operatorname{Cone}\left(\alpha, \mathcal{F}, s_{0}\right)$ all reals $r>1$ have colour 0 , and in the asymptotic cone $\operatorname{Cone}\left(\alpha, \mathcal{F}, s_{1}\right)$ all 
reals $r>1$ have colour 1 only. These two metric spaces are clearly not quasi-isometric.

Define $p_{n}$ and $q_{n}$, inductively, by: $p_{1}=q_{1}=1$, and

$$
\begin{aligned}
p_{n+1} & =n \sum_{i=1}^{n}\left(p_{i}+q_{i}\right) \\
q_{n+1} & =n\left(\sum_{i=1}^{n}\left(p_{i}+q_{i}\right)+p_{n+1}\right)
\end{aligned}
$$

Now consider the string $\alpha=0^{p_{1}} 1^{q_{1}} 0^{p_{2}} 1^{q_{2}} \ldots$, and define the following two scale functions:

- $s_{0}(n)=\sum_{i=1}^{n}\left(p_{i}+q_{i}\right)$, and

- $s_{1}(n)=\left(\sum_{i=1}^{n}\left(p_{i}+q_{i}\right)+p_{n+1}\right)$.

Also let $d_{j, n}(x, y)=|y-x| / s_{j}(n)(j=0,1)$. Then all points $a$ in $\left(\alpha, d_{j, n}\right)$ such that $1<d_{j, n}(0, a)<n$ have the colour $j$. Hence for any $r>1$, the colour of $r$ is 0 in $\operatorname{Cone}\left(\alpha, \mathcal{F}, s_{0}\right)$ and 1 in $\operatorname{Cone}\left(\alpha, \mathcal{F}, s_{1}\right)$.

The next theorem shows that the asymptotic cones of all Martin-Löf random strings [11] coincide when the scaling factor is a computable function.

Theorem VI.6. If $\alpha$ is Martin-Löf random, then for all computable scaling factors $s$ and ultra-filters $\mathcal{F}$, the asymptotic cone Cone $(\alpha, \mathcal{F}, s)$ coincides with the space $\left(\mathcal{R}_{\geqslant 0} ; d, C\right)$, where every real has all colours from $\Sigma$.

Proof. We assume that $\Sigma=\{0,1\}$. For given $r \in \mathbb{R}_{\geqslant 0}$, assume the following:

$\exists A \in \mathcal{F} \forall \epsilon \forall^{\infty} n \in A \exists r^{\prime} \in \alpha_{n}\left[r-\epsilon \leqslant r^{\prime} \leqslant r+\epsilon \wedge C_{n}\left(r^{\prime}\right)=0\right]$.

Then we have a sequence $\left(a_{n}\right)$ such that the subsequence $\left\{a_{n} \mid n \in A\right\}$ converges to $r$ and all elements of the subsequence are coloured by 0 , and hence $\operatorname{Cone}(\alpha, \mathcal{F}, s)$ has the colour 0 at $r$.

Taking the contraposition of this fact and letting $A=\mathbb{N}$, we have the following: if $\operatorname{Cone}(\alpha, \mathcal{F}, s)$ does not have the colour 0 at $r$ (by virtue of Lemma VI.2 we can assume that $r \in \mathbb{Q} \geqslant 0$ ), then for some $\epsilon>0$ we have infinitely many $n \in \mathbb{N}$ such that all points $r^{\prime}$ of $\alpha_{n}$ in an interval $[r-\epsilon, r+\epsilon]$ has the colour 1 .

Now assume such an $r$ exists and consider the set:

$G_{n}=\left\{X \in 2^{\omega} \mid s(n)(r-\epsilon) \leqslant i \leqslant s(n)(r+\epsilon) \Rightarrow X(i)=1\right\}$

In the Cantor space $\{0,1\}^{\omega}$, the sequence of open sets $\left(G_{n}\right)$ is uniformly computably enumerable and the sum of the measures of $G_{n}$ is bounded. Such sequences are called Solovay tests. This Solovay test fails the string $\alpha$, that is, $\alpha \in G_{n}$ for infinitely many $n$ (cf. Definition 3.2.18 in [11]). It is known that falling Solovay tests is equivalent failing MartinLöf tests (cf. Proposition 3.2.19 in [11]). This shows that $\alpha$ is not random.

A culmination of asymptotic cones construction is that $[\alpha]=[\beta]$ implies bi-Lipschitz equivalence between Cone $(\alpha, \mathcal{F}, s)$ and $\operatorname{Cone}(\beta, \mathcal{F}, s)$. The proof is standard with the difference that our metric spaces are coloured. However, since our base metric space is a linear order $\omega$, the conclusion of our theorem is a little stronger as it implies order preservance.

Theorem VI.7. If strings $\alpha$ and $\beta$ have the same large scale geometry then there are colour-preserving and order preserving homeomorphisms

$$
\begin{gathered}
H: \text { Cone }(\alpha, \mathcal{F}, s) \rightarrow \text { Cone }(\beta, \mathcal{F}, s) \text { and } \\
G: \text { Cone }(\beta, \mathcal{F}, s) \rightarrow \operatorname{Cone}(\alpha, \mathcal{F}, s),
\end{gathered}
$$

constants $C_{H}, C_{G}$ such that for all $\mathbf{a}, \mathbf{b} \in \operatorname{Cone}(\alpha, \mathcal{F}, s)$ and $\mathbf{c}, \mathbf{d} \in \operatorname{Cone}(\beta, \mathcal{F}, s)$ we have:

$$
\begin{gathered}
\left(1 / C_{H}\right) \cdot D(\mathbf{a}, \mathbf{b}) \leqslant D(H(\mathbf{a}), H(\mathbf{b})) \leqslant C_{H} \cdot D(\mathbf{a}, \mathbf{b}) \\
\quad \text { and } \\
\left(1 / C_{G}\right) \cdot D(\mathbf{c}, \mathbf{d}) \leqslant D(G(\mathbf{c}), G(\mathbf{d})) \leqslant C_{G} \cdot D(\mathbf{c}, \mathbf{d}) .
\end{gathered}
$$

\section{ACKNOWLEDGMENT}

This work was supported by JST ERATO Grant Number JPMJER1603, Japan. The authors thank Kazushige Terui for discussions. The first author thanks JSPS and RIMS of Kyoto University for support.

\section{REFERENCES}

[1] B. Velickovic and T. Simon. Asymptotic cones of finitely generated groups. Bulletin of the London Mathematical Society, vol. 32, pp. 203-220 (2000).

[2] A Dioubina, I. Polterovich, Explicit constructions of universal R-trees and asymptotic geometry of hyperbolic spaces, preprint, math.DG/9904133

[3] A Dioubina, I. Polterovich, Structures at infinity of hyperbolic spaces, (Russian) Uspekhi Mat. Nauk 53 (1998), no. 5(323), 239240; translation in Russian Math. Surveys 53 (1998), no. 5, 10931094.

[4] L. Van Den Dries and A. Wilkie. On Gromov's theorem concerning groups of polynomial growth and elementary logic, Journ. of Algebra 89 (1984), 349-374.

[5] C. Drutu, M. Kapovich. Lectures on geometric group theory. Preprint, 500p. Version: June 2016. The AMS series "Colloquium Publications". To be published, 2017.

[6] M.Gromov, Groups of polynomial growth and expanding maps : Inst. Hautes Etudes Sci. Publ. Math. No. 53 (1981) 5375.

[7] M.Gromov, Hyperbolic groups : in Essays in Group Theory(ed. S.M.Gersten) M.S.R.I. Publications No. 8, SpringerVerlag (1987) 75263.

[8] M.Gromov, Asymptotic invariants of infinite groups: in Geometric group theory, Vol. (eds. G.A.Niblo, M.A.Roller) London Math. Soc. Lecture Notes Series No. 182, Cambridge Univ. Press (1993) 1295.

[9] P. de la Harpe, Topics in geometric group theory: Chicago Lectures in Mathematics, University of Chicago Press (2000).

[10] C. G. Jockusch and R. Soare. $\Pi_{1}^{0}$-classes and degrees of theories. Transactions of the American Mathematical Society, 173, p. 3356, 1972.

[11] A. Nies. Computability and Randomness. Oxford University Press, 2012.

[12] H. Rogers. Theory of Recursive Functions and Effective Computability. MIT Press, 1987. 